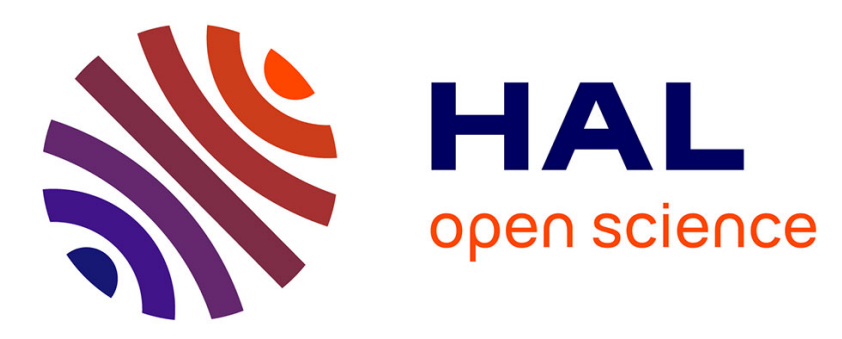

\title{
Computer-Aided Detection and diagnosis for prostate cancer based on mono and multi-parametric MRI: A review
}

Guillaume Lemaître, Robert Martí, Jordi Freixenet, Joan C. Vilanova, Paul M. Walker, Fabrice Meriaudeau

\section{To cite this version:}

Guillaume Lemaître, Robert Martí, Jordi Freixenet, Joan C. Vilanova, Paul M. Walker, et al.. Computer-Aided Detection and diagnosis for prostate cancer based on mono and multi-parametric MRI: A review. Computers in Biology and Medicine, 2015, 60, 10.1016/j.compbiomed.2015.02.009 . hal-01235868

\section{HAL Id: hal-01235868 https://u-bourgogne.hal.science/hal-01235868}

Submitted on 30 Nov 2015

HAL is a multi-disciplinary open access archive for the deposit and dissemination of scientific research documents, whether they are published or not. The documents may come from teaching and research institutions in France or abroad, or from public or private research centers.
L'archive ouverte pluridisciplinaire HAL, est destinée au dépôt et à la diffusion de documents scientifiques de niveau recherche, publiés ou non, émanant des établissements d'enseignement et de recherche français ou étrangers, des laboratoires publics ou privés. 


\title{
Computer-Aided Detection and Diagnosis for prostate cancer based on mono and multi-parametric MRI: A review
}

\author{
Guillaume Lemaître $^{\mathrm{a}, \mathrm{c}, *}$, Robert Martí ${ }^{\mathrm{c}}$, Jordi Freixenet ${ }^{\mathrm{c}}$, Joan C. Vilanova $^{\mathrm{d}}$, Paul M. Walker ${ }^{\mathrm{b}}$, \\ Fabrice Meriaudeau ${ }^{\mathrm{a}}$ \\ ${ }^{a}$ LE2I-UMR CNRS 6306, Université de Bourgogne, 12 rue de la Fonderie, 71200 Le Creusot, France \\ ${ }^{b}$ LE2I-UMR CNRS 6306, Université de Bourgogne, Avenue Alain Savary, 21000 Dijon, France \\ ${ }^{c}$ ViCOROB, Universitat de Girona, Campus Montilivi, Edifici P4, 17071 Girona, Spain \\ ${ }^{d}$ Department of Magnetic Resonance, Clinica Girona, Lorenzana 36, 17002 Girona, Spain
}

\begin{abstract}
Prostate cancer is the second most diagnosed cancer of men all over the world. In the last decades, new imaging techniques based on Magnetic Resonance Imaging (MRI) have been developed improving diagnosis. In practise, diagnosis can be affected by multiple factors such as observer variability and visibility and complexity of the lesions. In this regard, computer-aided detection and computer-aided diagnosis systems have been designed to help radiologists in their clinical practice. Research on computer-aided systems specifically focused for prostate cancer is a young technology and has been part of a dynamic field of research for the last ten years. This survey aims to provide a comprehensive review of the state of the art in this lapse of time, focusing on the different stages composing the work-flow of a computer-aided system. We also provide a comparison between studies and a discussion about the potential avenues for future research. In addition, this paper presents a new public online dataset which is made available to the research community with the aim of providing a common evaluation framework to overcome some of the current limitations identified in this survey.
\end{abstract}

Keywords: computer-aided detection, computer-aided diagnosis, prostate cancer, magnetic resonance imaging, magnetic resonance spectroscopy imaging, computer vision

\section{Introduction}

During the last century, physicists have focused on constantly innovating in terms of imaging techniques assisting radiologists to improve cancer detection and diagnosis. However, human diagnosis still suffers from low repeatability, synonymous with erroneous detection or interpretations of abnormalities throughout clinical decisions $[1,2]$. These errors are driven by two majors causes [1]: observer limitations (e.g.,

\footnotetext{
* Corresponding author.

Email addresses: guillaume.lemaitre@udg.edu (Guillaume Lemaître), marly@eia.udg.edu (Robert Martí), jordif@eia.udg.edu (Jordi Freixenet), pwalker@u-bourgogne.fr (Paul M. Walker), fabrice.meriaudeau@u-bourgogne.fr (Fabrice Meriaudeau)
} 
constrained human visual perception, fatigue or distraction) and the complexity of the clinical cases themselves, for instance due to unbalanced data (number of healthy cases more abundant than malignant cases) or overlapping structures.

Computer vision has given rise to many promising solutions, but, instead of focusing on fully automatic computerized systems, researchers have aimed at providing computer image analysis techniques to aid radiologists in their clinical decisions [1]. In fact, these investigations brought about both concepts of Computer-Aided Detection (CADe) and Computer-Aided Diagnosis (CADx) grouped under the acronym CAD. Since those first steps, evidence has shown that CAD systems enhance the diagnosis performance of radiologists. Chan et al. reported a significant $4 \%$ improvement in breast cancer detection [3], which has been confirmed in later studies [4]. Similar conclusions were drawn in the case of lung nodule detection [5], colon cancer [6] and Prostate Cancer (CaP) as well [2]. Chan et al. also hypothesized that CAD systems will be even more efficient assisting inexperienced radiologists than senior radiologists [3]. This hypothesis was tested by Hambrock et al. [2] and was confirmed in the case of CaP detection. In this particular study, inexperienced radiologists obtained equivalent performance to senior radiologists, both using CAD whereas the accuracy of their diagnosis was significantly poorer without CAD's help.

In contradiction with the aforementioned statement, $\mathrm{CAD}$ for $\mathrm{CaP}$ is a young technology due to the fact that it is based on Magnetic Resonance Imaging (MRI) [7]. Four distinct MRI modalities are employed in CaP diagnosis which were mainly developed after the mid-1990s: (i) $\mathrm{T}_{2}$ Weighted ( $\mathrm{T}_{2}-\mathrm{W}$ ) MRI [8], (ii) Dynamic Contrast-Enhanced (DCE) MRI [9], (iii) Magnetic Resonance Spectroscopy Imaging (MRSI) [10] and (iv) Diffusion Weighted (DW) MRI [11]. In addition, the increase of magnetic field strength (from 1.5 to 3 Tesla) and the development of endorectal coils, both improved image spatial resolution [12] needed to perform more accurate diagnosis. It is for this matter that the development of $\mathrm{CAD}$ for $\mathrm{CaP}$ is still lagging behind the other fields stated above.

The first study on CAD for MRI was published in 2003 by Chan et al. [13]. Despite this, no less than fifty studies have been reviewed for this survey since that seminal work. To the best of our knowledge, there is no fully detailed review in the literature regarding the advancement of CAD systems devoted specifically to CaP detection and diagnosis. Only the recent work of [14] briefly covers a reduced number of current research works on $\mathrm{CAD}$ for $\mathrm{CaP}$ (a total of 70 references), but it does not provide a detailed description of any of the steps of a CAD system as it is done here with more than 200 references. Moreover, a new categorisation is proposed in this work taking the clinical and technical aspects of a CAD system into account. Thus, the aim of this survey is threefold: (i) provide an overview and categorisation of the developed CAD systems for CaP detection and diagnosis based on MRI modalities (ii) assess the different works and (iii) point out avenues for future directions.

We also would like to emphasize the fact that this study will review the details of the computer vision aspects of the different studies. Thus, this survey is more intended for a medical imaging audience rather 
than a purely experimented clinical audience.

As discussed further in Sect. 2.2.3, we identified and characterized a common framework regarding the CAD systems. Stages involved in CAD work-flow can be categorized into three distinctive processes: (i) image regularization, (ii) CADe, (iii) CADx (see Fig. 1). Image regularisation focuses on formatting the data while CADe and CADx allow to detect possible lesions and distinguish malignant from non-malignant tumours, respectively.

This paper is organized as follows: Sect. 2 deals with general information about human prostate and background about CaP. Methods regarding CaP screening and imaging techniques used are also presented as well as an introduction to the CAD framework. Sect.3-4 review techniques used in different steps involved in a CAD work-flow which will be our main contribution. Image regularization framework including pre-processing (Sect. 3.1), segmentation (Sect. 3.2) and registration (Sect.3.3) will be covered as well as CADe and CADx strategies (Sect.4) identified. Results and discussion are reported in Sect. 5 followed by a concluding section. Deriving from this discussion, we make available to the research community a public online dataset aiming at overcoming some of the drawbacks found when evaluating research in this field.

\section{Background}

This section provides an overview of $\mathrm{CaP}$ as well as its detection and diagnosis. MRI plays an important role in improving the current strategy and a more detailed description of MRI modalities is given. Furthermore, a discussion regarding the aim of CAD systems is also given.

\subsection{Prostate carcinoma}

$\mathrm{CaP}$ has been reported on a worldwide scale to be the second most frequently diagnosed cancer of men accounting for $13.6 \%$ [15]. Statistically, in 2008, the number of new diagnosed cases was estimated to be 899, 000 with no less than 258, 100 deaths [15]. In United States, aside from skin cancer, CaP was declared to be the most commonly diagnosed cancer among men, implying that approximately one in six men will be diagnosed with $\mathrm{CaP}$ during their lifetime and one in thirty-six will die from this disease causing $\mathrm{CaP}$ to be the second most common cause of cancer death among men [16, 17].

Despite active research to determine the causes of prostate cancer, a fuzzy list of risk factors has been established [18]. The etiology was linked to the following factors [18]: (i) family history [19, 20], (ii) genetic factors [21, 22, 23], (iii) race-ethnicity [19, 24], (iv) diet [19, 25, 26], and (v) obesity [19, 27]. This list of risk factors alone cannot be used to diagnose $\mathrm{CaP}$ and in this way, screening enables early detection and treatment.

$\mathrm{CaP}$ growth is characterized by two main types of evolution [28]: slow and fast. The slow-growing tumours, accounting for up to $85 \%$ of all CaPs [29], progress slowly and usually stay confined to the 
prostate gland. For such cases, treatment can be substituted with active surveillance. In contrast, the second variant of $\mathrm{CaPs}$ develops rapidly and metastasises from prostate gland to other organs, primarily the bones [30]. Bone metastases, being an incurable disease, significantly affect the morbidity and mortality rate [31]. Hence, the results of the surveillance have to be trustworthy in order to distinguish aggressive from slow-growing $\mathrm{CaP}$.

$\mathrm{CaP}$ is more likely to develop in specific regions of the prostate. In that respect, around $70-80 \%$ of CaPs originate in Peripheral Zone (PZ) whereas 10-20 \% in Transitional Zone (TZ) [32, 33, 34]. Only about 5 $\%$ of CaPs occur in Central Zone (CZ) $[33,35]$. However, those cancers appear to be more aggressive and more likely to invade other organs due to their location [35].

\subsection{CaP screening and imaging techniques}

\subsubsection{Current CaP screening}

Current $\mathrm{CaP}$ screening consists of three different stages. First, Prostate-Specific Antigen (PSA) control is performed to distinguish between low and high risk CaP. Then, for confirmation, samples are taken during Transrectal UltraSound (TRUS) biopsy of the prostate and finally analysed to evaluate the prognosis and the stage of CaP. Although PSA screening has been shown to improve early detection of CaP [36], its lack of reliability motivates further investigations using MRI [37, 38, 39].

Hence, new screening methods should be developed with improved specificity of detection as well as more accurate risk assessment (aggressiveness and progression). Current research is focused on identifying new biological markers to replace PSA-based screening [40, 41, 42]. Until such research comes to fruition, these needs can be met through active-surveillance strategy using multi-parametric MRI techniques [43, 44]. A CAD system based on MRI, which is an area of active research and forms the focus of this paper, can be incorporated into this screening strategy allowing a more systematic and rigorous follow-up.

Another weakness of the current screening strategy lies in the fact that TRUS biopsy does not provide trustworthy results. Due to its "blind" nature imposed by the a random sampling strategy, there is a chance of missing aggressive tumours or detecting microfocal "cancers", which influences the aggressivenessassessment [45]. As a consequence, over-diagnosis is estimated at up to $30 \%$ [46], while missing clinically significant $\mathrm{CaP}$ is estimated at up to $35 \%$ [47]. In an effort to solve both issues, alternative biopsy approaches have been explored. MRI/UltraSound (US)-guided biopsy has been shown to outperform standard TRUS biopsy [48]. There, multimodal MRI images are fused with US images in order to improve localization and aggressiveness assessment to carry out biopsies. Human interaction plays a major role in biopsy sampling which can lead to low repeatability; by reducing potential human errors at this stage, the CAD framework can be used to improve repeatability of examination.

$\mathrm{CaP}$ detection and diagnosis benefit from the use of CAD and MRI techniques. In the following sections, these techniques will be presented in addition to an overview of CAD for CaP. 


\subsubsection{MRI imaging techniques}

Unlike TRUS biopsy, 3.0 Tesla MRI examination is a non-invasive protocol and has been shown to be the most accurate and harmless technique currently available [49]. In this section, we review different MRI modalities developed for CaP detection and diagnosis. Features used by the radiologists in their daily diagnosis task will receive particular attention together with their drawbacks. Moreover, these features commonly form the basis for developing analytic tools and automatic algorithms. However, we refer the reader to Sect. 4.2 for more details on automatic feature detection methods since they are part and parcel of the CAD framework. An exhaustive review regarding the different modalities as well as the characteristic of each of them is presented in [50].

$\mathrm{T}_{2}$-W MRI was the first MRI sequence used to perform CaP diagnosis using MRI [8]. Nowadays is a common practice for $\mathrm{CaP}$ detection, localization and staging. This imaging technique is well suited to render zonal anatomy of the prostate [50]. The features representative of $\mathrm{CaP}$ are indicated in Table 1. $\mathrm{PZ}$ and Central Gland (CG) tissues are well perceptible in these images. The former is characterized by an intermediate/high-Signal Intensity (SI) while the latter is depicted by a low-SI [51]. An example of a healthy prostate is shown in Fig. 2(a). In PZ, round or ill-defined low-SI masses are synonymous with CaPs [8] as shown in Fig. 2(b). Detecting CaP in CG is more challenging because both normal CG tissue and malignant tissue, have a low-SI in $\mathrm{T}_{2}$-W MRI reinforcing difficulties to distinguish between them. However, CaPs in CG appear often as homogeneous mass possessing ill-defined edges with lenticular or "water-drop" shapes $[52,50]$ as depicted in Fig. 2(c). CaP aggressiveness was shown to be inversely correlated with SI. Indeed, CaPs assessed with a Gleason Score (GS) of 4-5 implied lower SI than the one with a GS of 2-3 [53]. In spite of the usefulness of these features, the $\mathrm{T}_{2}$-W modality lacks reliability in some aspects [54, 43]. Sensitivity is affected by the difficulties in detecting cancers in CG [54] while specificity rate is highly affected by outliers $[51,55,11,56,50]$.

However, $\mathrm{T}_{2}$ values alone have been shown to be more discriminative [57] and highly correlated with citrate concentration, a biological marker in CaP [58, 59]. The Fast Spin-Echo (FSE) sequence has been shown to be particularly well suited in order to build a $\mathrm{T}_{2}$ map and obtain accurate $\mathrm{T}_{2}$ values [60]. Similar to $\mathrm{T}_{2}-\mathrm{W}$ MRI, $\mathrm{T}_{2}$ values associated with $\mathrm{CaP}$ are significantly lower than those of healthy tissues $[58,61]$.

DCE MRI is an imaging technique which exploits the vascularity characteristic of tissues [62]. Contrast media, usually gadolinium-based, is injected intravenously into the patient. The media extravasates from vessels to the Extravascular-Extracellular Space (EES) and is then released back into the vasculature before being eliminated by the kidneys [63]. Furthermore, the diffusion speed of the contrast agent may vary due to several parameters: (i) the permeability of the micro-vessels, (ii) their surface area and (iii) the blood flow [64]. DCE MRI is based on an acquisition of a set of $\mathrm{T}_{1}$ Weighted $\left(\mathrm{T}_{1}-\mathrm{W}\right) \mathrm{MRI}$ images over time. The Gadolinium-based contrast agent shortens $\mathrm{T}_{1}$ relaxation time enhancing contrast in $\mathrm{T}_{1}-\mathrm{W} \mathrm{MRI}$ images. The 
aim is to post-analyse the pharmacokinetic behaviour of the contrast media concentration in prostate tissues [62]. The image analysis is carried out in two dimensions: (i) in the spatial domain on a pixel-by-pixel basis and (ii) in the time domain corresponding to the consecutive images acquired with the MRI. Thus, for each spatial location, a signal linked to contrast media concentration is measured as shown in Fig. 3 [65]. As depicted in Fig. 3(b), CaPs are characterized by a signal having an earlier and faster enhancement as well as an earlier wash-out (cf., the rate of the contrast agent flowing out of the tissue) [62].

Three different approaches exist to analyse these signals with the aim of tagging them as corresponding to either normal or malignant tissues: qualitative analysis is based on assessment of the signal shape [43]; quantitative approaches consist of inferring pharmocokinetic parameter values [65]; and semi-quantitative methods which rely on shape characterization using mathematical modelling to extract a set of parameters [43, 62]. It was shown that semi-quantitative and quantitative methods improve localization of $\mathrm{CaP}$ when compared with qualitative methods [66]. Table 1 gives an overview of the features used during DCE MRI analysis. Section 4.2.2 provides a full description of quantitative and semi-quantitative approaches. DCE MRI combined with $\mathrm{T}_{2}$-W MRI has shown to enhance sensitivity compared to $\mathrm{T}_{2}$-W MRI alone $[67,68,69,70]$. Despite this fact, DCE MRI possesses some drawbacks. Due to its "dynamic" nature, patient motions during the image acquisition may lead to spatial misregistration of the image set [62]. Furthermore, it has been suggested that malignant tumours are difficult to distinguish from prostatitis located in PZ and Benign Prostatic Hyperplasia (BPH) located in CG [43, 62] as these two pairs of tissues tend to have similar appearances. Later studies have shown that CaPs in CG do not always manifest in homogeneous fashion. Indeed, tumours in this zone can present both hypo-vascularization and hyper-vascularization which illustrates the challenge of CaP detection in CG [71].

DW MRI is the most recent MRI imaging technique aiming at CaP detection and diagnosis [11]. This modality exploits the variations in the motion of water molecules in different tissues [72, 73]. Table 1 summarizes the markers used in DW MRI to distinguish CaP. From the Nuclear Magnetic Resonance (NMR) principle side, DW MRI sequence produces contrasted images due to variation of water molecules motion. The method is based on the fact that the signal in DW MRI images is inversely correlated to the degree of random motion of water molecules [74]. A higher degree of random motion results in a more significant signal loss whereas a lower degree of random motion is synonymous with lower signal loss [74]. Under these conditions, the MRI signal is measured as:

$$
\begin{gathered}
M_{x, y}(t, b)=M_{x, y}(0) \exp \left(-\frac{t}{\mathrm{~T}_{2}}\right) S_{\mathrm{ADC}}(b), \\
S_{\mathrm{ADC}}(b)=\exp (-b \times \mathrm{ADC})
\end{gathered}
$$

where $S_{\mathrm{ADC}}$ refers to signal drop due to diffusion effect, ADC is the Apparent Diffusion Coefficient and $b$ 
is the attenuation coefficient depending only on gradient pulses parameters: (i) gradient intensity and (ii) gradient duration [75].

By using this formulation, image acquisition with a parameter $b=0 \mathrm{~s} \cdot \mathrm{mm}^{-2}$ corresponds to a $\mathrm{T}_{1}-\mathrm{W}$ MRI acquisition. Then, increasing the attenuation coefficient $b$ (cf., increase gradient intensity and duration) enhances the contrast in DW MRI images. To summarize, in DW MRI images, CaPs are characterized by high-SI compared to normal tissues in PZ and CG as shown in Fig.4(a) [50]. However, some tissues in CG can look similar to CaP with higher SI [50]. Diagnosis using DW MRI combined with $\mathrm{T}_{2}$-W MRI has shown a significant improvement compared with $\mathrm{T}_{2}-\mathrm{W}$ MRI alone and provides highly contrasted images [76, 77, 78]. As drawbacks, this modality suffers from poor spatial resolution and low specificity [78].

With a view to eliminate these drawbacks, radiologists are extracting quantitative maps from DW MRI which is known as the ADC map. The ADC coefficient is considered as a "pure" diffusion coefficient. From Eq. 1, it is clear that performing multiple acquisitions only varying $b$ will not have any effect on the term $M_{x, y}(0) \exp \left(-\frac{t}{T_{2}}\right)$. Thus, Eq. 1 can be rewritten as:

$$
S(b)=S_{0} \exp (-b \times \mathrm{ADC}) .
$$

To compute the ADC map, a minimum of two acquisitions are necessary: (i) for $b_{0}=0 \mathrm{~s} \cdot \mathrm{mm}^{-2}$ where the measured signal is equal to $S_{0}$, and (ii) $b_{1}>0 \mathrm{~s} . \mathrm{mm}^{-2}$ (typically $1000 \mathrm{~s} . \mathrm{mm}^{-2}$ ). Then, the ADC map can be computed as:

$$
\mathrm{ADC}=-\frac{\ln \left(\frac{S\left(b_{1}\right)}{S_{0}}\right)}{b_{1}} .
$$

More accurate computation of the ADC map can be obtained by performing several acquisitions with different values for the parameter $b$ and performing a semi-logarithmic linear fitting using the model presented in Eq. (3). Regarding the appearance of the ADC maps, it was previously stated that by increasing the value of $b$, the signal of CaP tissue increases significantly. From Eq. (4), it can be shown that tissue appearance in the ADC map will be the inverse of DW MRI images. This coefficient varies inversely to DW MRI images [50] as depicted in Fig. 4(b). Similar to the gain achieved by DW MRI, diagnosis using ADC map combined with $\mathrm{T}_{2}$-W MRI significantly outperforms $\mathrm{T}_{2}-\mathrm{W}$ MRI alone [79, 78]. Moreover, it has been shown that ADC is correlated with GS $[80,81,82]$. However, some tissues of the CG zone mimic CaP with low-SI [54] and image distortion can arise due to haemorrhage [78]. It has also been noted that a high variability of the ADC occurs between different patients making it difficult to define a static threshold to distinguish CaP from non-malignant tumours [78].

$\mathrm{CaP}$ induces metabolic changes in the prostate compared with healthy tissue. Thus, CaP detection can be carried out by tracking changes of metabolite concentration in prostate tissue. MRSI is an NMR-based 
technique which generates spectra of relative metabolite concentration in a Region Of Interest (ROI). In order to track changes of metabolite concentration, it is important to know which metabolites are associated with $\mathrm{CaP}$. To address this question, clinical studies identified three biological markers: (i) citrate, (ii) choline and (iii) polyamines composed mainly of spermine, and in less abundance of spermidine and putrescine [83, $84,85]$.

An increased concentration of choline associated with a decreased concentration of citrate and spermine are related to the presence of $\mathrm{CaP}[83,84,86,85]$. To determine the concentration of these biological markers, one has to focus on the MRSI modality. In each spectrum acquired, each peak is associated with a particular metabolite and the area under each peak corresponds to the relative concentration of this metabolite (see Fig. 5) [87]. Hence, frequencies of interest in regard to $\mathrm{CaP}$ detection and diagnosis should correspond to the earlier mentioned metabolites. Choline and spermine are represented by a single peak at respectively 3.21 ppm and $3.11 \mathrm{ppm}$ [88]. Due to the coupling effect, citrate is represented by three or four peaks depending on the magnetic field strength. Citrate ranges from $2.47 \mathrm{ppm}$ to $2.81 \mathrm{ppm}$ with a central frequency at 2.64 ppm [88]. Then, relative concentrations of these metabolites are obtained by computing the area under the curve of the spectrum between the lower and upper frequency limits of each peak (see Fig. 5). It can be noted that a creatine peak is located at $3.02 \mathrm{ppm}$ and the three metabolite peaks tend to be merged together at clinical magnetic field strengths (see Fig. 5) [43, 86].

The variations of the metabolites are reported in Table 1. MRSI allows examination with high specificity and sensitivity compared to other MRI modalities [78]. Furthermore, it has been shown that combining MRSI with MRI improves detection and diagnosis performance [89, 90, 91]. Citrate and spermine concentrations are inversely correlated with the GS allowing to distinguish low from high grade CaPs [85]. However, choline concentration does not provide the same properties [85]. Unfortunately, MRSI also presents several drawbacks. First, MRSI acquisition is time consuming which prevents this modality from being used in daily clinical practise [50]. In addition, MRSI suffers from low spatial resolution due to the fact that Signalto-Noise (SNR) is linked to the voxel size. However, this issue is addressed by developing new scanners with higher magnetic field strengths such as $7.5 \mathrm{~T}$ [85]. Finally, a high variability of the relative concentrations between patients has been observed [78]. The same observation was made depending on the zones studied (cf., PZ, CG, base, mid-gland, apex) [92, 93]. Due to this variability, it is difficult to use a fixed threshold in order to differentiate $\mathrm{CaP}$ from healthy tissue.

\subsubsection{Computer-aided systems for CaP: $C A D e-C A D x$}

As previously mentioned in the introduction (see Sect.1), CADs are developed to advise and backup radiologists in their tasks of $\mathrm{CaP}$ detection and diagnosis, but not to provide fully automatic decisions [1]. CADs can be divided into two different sub-groups either as CADe, with the purpose to highlight probable lesions in MRI images, or CADx, which focuses on differentiating malignant from non-malignant tumours [1]. 
Moreover, an intuitive approach, motivated by developing a framework combining detection-diagnosis, is to mix both CADe and CADx by using the output of the former mentioned as a input of the latter named. Although the outcomes of these two systems should differ, the framework of both CAD systems is similar. A general CAD work-flow is presented in Fig. 1.

MRI modalities mentioned in Sect. 2.2.2 are used as inputs of CAD for CaP. The images acquired from the different modalities show a large variability between patients: the prostate organ can be located at different positions in images (e.g., patient motion, variation of acquisition plan), and the SI can be corrupted with noise or artefacts during the acquisition process (eg., magnetic field inhomogeneity, use of endorectal coil). To address these issues, the first stage of CAD is to pre-process multiparametric MRI images to reduce noise, remove artefacts and standardize the SI. As most of the later processes will be only focused on the prostate. It is necessary to segment the prostate in each MRI-modality to define it as a ROI. However, data may suffer from misalignment due to patient motion or different acquisition parameters. Therefore, a registration step is usually performed so that all the previously segmented MRI images will be in the same reference frame. Registration and segmentation steps can be swapped depending on the strategy chosen.

Some studies do not fully apply the methodology depicted in Fig. 1. Details about those can be found in Table 2. Some studies proposed methods in which inputs are the MRI raw data in order to demonstrate the robustness of their approaches to noise or artefacts. In some cases, prostate segmentation is performed manually as well as registration. It is also sometimes assumed that no patient motions occur during the acquisition procedure, removing the need of registering the multiparametric MRI images.

Once the data are regularized, it becomes possible to extract features and classify these data to obtain either the location of possible lesions (CADe) or/and the malignancy nature of these lesions (CADx).

In a CADe framework, possible lesions will be segmented automatically and further used as inputs of a CADx. Nevertheless, some works also used a fused CADe-CADx framework in which a voxel-based features are directly used, allowing to obtain the location of the malignant lesions as results. On the other hand, manual lesions segmentation is not considered to be part of a CADe.

CADx is composed of the processes allowing to distinguish malignant from non-malignant tumours. In the studies reviewed, CaP malignancy is defined using the grade of the GS determined after postbiopsy or prostatectomy. As presented in Fig. 1, CADx is usually composed of the three common steps used in classification framework: (i) features detection, (ii) features extraction/selection and (iii) features classification.

\subsection{Literature classification}

The CAD review is organized using the methodology presented in Fig. 1. Methods embedded in the image regularization framework are presented initially to subsequently focus on the image classification framework, being divided into CADe and CADx. Table 2 summarizes the forty-two different CAD studies 
reviewed in this paper. The first set of information reported is linked to the data acquisition such as the number of patients included in the study, the modalities acquired as well as the strength of the field of the scanner used. Subsequently, information about the prostate zones considered in the CAD analysis (PZ or $\mathrm{CG}$ ) are reported since that detecting $\mathrm{CaP}$ in the $\mathrm{CG}$ is a more challenging problem and has received particular attention only in recent publications.

\section{Image regularization framework}

This section provides a review of the methods used in CADs for $\mathrm{CaP}$ in order to regularize input images. We start with pre-processing methods presented in Sect.3.1, focusing mainly on the reduction of noise level and artefacts as well as standardization of SI. Section 3.2 and Sect. 3.3 will be dedicated to segmentation methods, so that later methods only operate on the segmented prostate, and registration to align segmented images from different MRI-modalities in the same reference frame.

\subsection{Pre-processing}

\subsubsection{MRI images pre-processing}

Three different groups of pre-processing methods are commonly applied to images as initial stage in CAD for CaP.

- Noise filtering: The NMR signal measured and recorded in the k-space during an MRI acquisition is affected by noise. This noise obeys a complex Gaussian white noise mainly due to thermal noises in the patient area [94]. Furthermore, MRI images visualized by radiologists are in fact the magnitude images resulting from the complex Fourier transform of the k-space data. The complex Fourier transform, being a linear and orthogonal transform, does not affect the Gaussian noise characteristics [94]. However, the function involved in the magnitude computation is a non-linear transform (i.e., the square root of the sum of squares of real and the imaginary parts), implying that the noise distribution is no longer Gaussian; it indeed follows a Rician distribution making the denoising task harder. Briefly, a Rician distribution can be characterized as follows: in low-SI region (low SNR), it can be approximated with a Rayleigh distribution while in high-SI region (high SNR), it is similar to a Gaussian distribution [95]. Reviews of all denoising methods can be found in [96, 97]. Median filtering is the simplest approach used to address the denoising issue in MRI images [98, 99]. However, from a theoretical point of view, this simple filtering method is not well formalized to address the noise distribution in MRI images. More complex approaches were proposed to overcome this problem. A common method used to denoise MRI images is based on wavelet-based filtering. Investigations focus on the strategies to perform the most adequate coefficient shrinkage method (e.g., using thresholding, singularity property or Bayesian framework) [100]. Ampeliotis et al. in $[101,102]$ performed wavelet shrinkage to denoise magnitude MRI images (cf., $\mathrm{T}_{2}$-W-MRI and 
DCE-MRI) using thresholding techniques [103]. However, since the wavelet transform is an orthogonal transform, the Rician distribution of the noise is preserved in the wavelet-domain. Hence, for low SNR, the wavelet and scaling coefficients still suffer from a bias due to this specific noise distribution [94]. Lopes et al. in [104] used the filtering technique proposed by [105] to denoise $\mathrm{T}_{2}$-W-MRI which was based on joint detection and estimation theory [106].

- Bias correction: Besides being corrupted by noise, MRI images are also affected by the inhomogeneity of the MRI field commonly referred to as bias field [107]. This bias field results in a smooth variation of the SI through the image. When an endorectal coil is used, an artefact resulting of an hyper-intense signal can be observed around the coil on the images. As a consequence, the SI of identical tissues varies depending on their spatial location in the image making further processes such as segmentation or registration harder $[108,109]$. A review of bias correction methods can be found in [109].

Viswanath et al. in [110] performed bias correction on $\mathrm{T}_{2}$-W-MRI using a parametric Legendre polynomial model proposed in [107] and available in the Insight Segmentation and Registration Toolkit (ITK) library ${ }^{1}$.

$\mathrm{Lv}$ et al. in [111] corrected the inhomogeneity in $\mathrm{T}_{2}$-W-MRI images by using the method proposed in [112]. In this method, the MRI images are corrected iteratively by successively detecting the image foreground via generalized scale ( $g$-scale) and estimating a bias field function based on a second-order polynomial model.

- SI normalization/standardization: As discussed in the later section, segmentation or classification tasks are usually performed by first learning from a training set of patients. Hence, one can emphasize the desire to perform MRI examinations with a high repeatability or in other words, one would like to obtain similar MRI images (cf., similar SIs) for patients of the same group (cf., healthy patients vs. patients with $\mathrm{CaP}$ ), for a similar sequence.

However, it is a known fact that variability between patients occurs during the MRI examinations even using the same scanner, protocol or sequence parameters [113]. Hence, the aim of normalization or standardization of the MRI data is to remove the variability between patients and enforce the repeatability of the MRI examinations. Approaches used to standardize MRI images can be either categorized as statistical-based standardization or organ SI-based standardization.

Artan et al. $[114,115]$ as well as Ozer et al. $[98,99]$ standardized $T_{2}-W$, DCE and DW MRI images by computing the standard score (also called $z$-score) of the pixels of the PZ. In a similar way, Liu et al. [116] normalized $\mathrm{T}_{2}$-W-MRI by making use of the median and interquartile range for all the pixels.

Lv et al. [111] scaled the SI of $\mathrm{T}_{2}$-W-MRI images using the method proposed in [117] based on

\footnotetext{
${ }^{1}$ The ITK library is available at: http://www.itk.org/
} 
Probability Density Function (PDF) matching. This approach is based on the assumption that MRI images from the same sequence should share the same PDF appearance. Hence, one can approach this issue by transforming and matching the PDFs using some statistical landmarks such as median and different quantiles.

Viswanath et al. in $[110,118,119]$ used a variant of this previous approach presented in [120] aiming to standardize the $\mathrm{T}_{2}$-W-MRI images. Instead of computing the PDF of an entire image, a pre-segmentation of the foreground is carried out via $g$-scale.

The methods described above were statistical-based methods. However, the standardization problem can be tackled by normalizing the MRI images using the SI of some known organs present in these images. Niaf et al. $[121,122]$ normalized $\mathrm{T}_{2}$-W-MRI images by dividing the original SI of the images by the mean SI of the bladder. Likewise, in [121] standardized the $\mathrm{T}_{1}$-W-MRI images using the Arterial Input Function (AIF) as proposed in [123].

\subsubsection{MRSI spectra}

Presented in Sect. 2.2.2, MRSI is a modality related to a one dimensional signal. Hence, specific preprocessing steps for this type of signals have been applied instead of standard signal processing methods.

- Phase correction: MRSI data acquired suffer from zero-order and first-order phase misalignments [124, 125]). Parfait et al. [126] used a method proposed in [124] where the phase of MRSI signal is corrected based on entropy minimization in the frequency domain.

- Water and lipid residuals filtering: The water and lipid metabolites occur in much higher concentrations than the metabolites of interests (cf., choline, creatine and citrate) $[127,125]$. Fortunately, specific MRSI sequences were developed in order to suppress water and lipid metabolites using pre-saturation techniques [127]. However, these techniques do not perfectly remove water and lipids peaks and some residuals are still present in the MRSI spectra. Therefore, different post-processing methods have been proposed to enhance the quality of the MRSI spectra by removing these residuals. For instance, Kelm et al. [128] used the well known HSVD algorithm proposed in [129] which models the MRSI signal by a sum of exponentially damped sinusoids in the time domain.

- Baseline correction: Sometimes, the problem discussed in the above section regarding the lipid molecules is not addressed simultaneously with water residuals suppression. Lipids and macromolecules are known to affect the baseline of the MRSI spectra. They could cause errors during further fitting processes aiming to quantify the metabolites, especially regarding the citrate metabolite.

Parfait et al. [126] made the comparison of two different methods to detect the baseline and correct the MRSI spectra based on [130, 131]. Liber et al. [130] addressed the problem of baseline detection in the frequency domain by iteratively fitting a polynomial of low degree whereas Parfait et al. [126] 
modified this algorithm by convolving a Gaussian kernel to smooth the MRSI signal instead of fitting a polynomial function. Unlike in [130], Devos et al. in [131] proposed to correct the baseline in the time domain by multiplying the MRSI signal by a decreasing exponential function. However, Parfait et al. [126] concluded that the method proposed in [130] outperformed the one in [131].

In the contemporary work of Tiwari et al. [132], the authors detected the baseline using a local nonlinear fitting method avoiding regions with significant peaks which were detected using a experimentally parametrised signal-to-noise ratio (i.e. a value larger than $5 \mathrm{~dB}$ ).

- Frequency alignment: Due to variations of the experimental conditions, a frequency shift can be observed in the MRSI spectra [124, 125]. Tiwari et al. [132] corrected the frequency shift by first detecting known metabolite peaks such as choline, creatine and citrate. The frequency shift is corrected by minimizing the frequency error between the experimental and theoretical values of each of these peaks.

- Normalization: Due to variations of the experimental conditions, the MRSI signal may also vary between patients. Parfait et al. [126] as in [131] compared two methods to normalize the MRSI signal. In each method, the original MRSI spectra is divided by a normalization factor, similar to the intensity normalization described earlier. The first approach to obtain the normalization factor is based on an estimation of the water concentration. It is required to have an additional MRSI sequence where the water metabolites are unsuppressed. Using this sequence, an estimation of the water concentration can be performed using the previously reported HSVD algorithm. The second approach to normalization is based on using the $\mathrm{L}_{2}$ norm of the MRSI spectra. It should be noted that both studies concluded that the $\mathrm{L}_{2}$ normalization was more efficient in their framework $[126,131]$.

\subsection{Segmentation}

The segmentation task consists of delineating the prostate boundaries in the MRI and is of particular importance for focusing the posterior processing on the organ of interest [133]. In this section, only the segmentation methods used in $\mathrm{CAD}$ for $\mathrm{CaP}$ systems are presented and summarized in Table 4, and are mostly intensity based. An exhaustive review of prostate segmentation methods in MRI can be found in [133] as well as prostate modelling in [134].

- Manual segmentation: To highlight the importance of prostate segmentation task in CAD systems, it is interesting to note the large number of studies which manually segment the prostate organs $[114,115$, $135,121,122,98,99,136,137,138]$. In all the cases, the boundaries of the prostate gland are manually defined in order to limit the further processing to only this area. This approach ensures the right delineation of the organ nevertheless this procedure is highly time consuming and should be performed by a radiologist. 
- Atlas-based segmentation: Litjens et al. in [139] used a multi-atlas-based segmentation [140] using multi-modal images (e.g., $\mathrm{T}_{2}$-W-MRI and ADC map) to segment the prostate with an additional pattern recognition method to differentiate $\mathrm{CG}$ and $\mathrm{PZ}$ as proposed in [141]. This method consists of three different steps: (i) the registration between each atlas and the multi-modal images, (ii) the atlas selection and finally (iii) the classification of the prostate segmented voxels in either CG or PZ.

Litjens et al. in [142] used an almost identical algorithm proposed in the PROMISE12 challenge [143]. Their segmentation method is also based on multi-atlas multi-modal images, but the SIMPLE method [144] is used instead to combine labels after the registration of the different atlas to obtain the final segmentation.

- Model-based segmentation: Viswanath et al. in $[145,110]$ used the Multi-Attribute Non-initializing Texture Reconstruction based Active shape model (MANTRA) method as proposed in [146]. MANTRA is closely related to the Active Shape Model (ASM) from [147]. This algorithm consists of two stages: (i) a training stage where a shape and appearance model is generated and (ii) the actual segmentation performed based on the learned model.

Litjens et al. [148] and Vos et al. [149] used an approach proposed in [150] in which the bladder, prostate and rectum are segmented. The segmentation task is performed as an optimization problem taking three parameters into account linked to organs such as: (i) the shape, (ii) the location and (iii) the respective angles between them. Furthermore, Litjens et al. [148] used only the ADC map to encode the appearance whereas Vos et al. [149] used both ADC and $\mathrm{T}_{2}$ maps.

Only the work of Tiwari et al. in [151] proposes a segmentation based on MRSI. Authors localized the voxels corresponding to the prostate organ using a hierarchical spectral clustering. First, each MRSI spectrum is projected into a lower dimension space using graph embedding [152].

\subsection{Registration}

The role of image registration is vital in CAD systems using multi-parametric MRI images. As it will be discussed in Sect. 4, for the sake of an optimal classification, the features detected in each modality will be grouped depending of their spatial locations. Hence, one has to ensure the perfect alignment of the multi-modal MRI images ahead of performing any classification.

Image registration is the procedure consisting of aligning an unregistered image (also called moving image) into a template image (also called fixed image) via a geometric transformation. This problem is usually addressed as presented in Fig. 6. An iterative procedure takes place to infer the geometric transformation (parametric or non-parametric) via an optimizer, which maximizes the similarity between the two images. From Sect.3.3.1 to Sect.3.3.4, we individually review the different components of a typical registration framework: transformation model, similarity metric, optimizer and interpolation. Section 3.3.5 
will summarize the combinations of these components especially used in $\mathrm{CAD}$ for $\mathrm{CaP}$ systems. Exhaustive reviews covering registration methods in computer science and medical fields can be found in [153, 154], respectively.

\subsubsection{Geometric transformation models}

From all CAD for CaP systems reviewed, only parametric transformation models have been used, mainly based on affine and elastic transformations. Affine transformations provide degrees of freedom managing rotations and translation as with the rigid transformations but also shearing and scaling. Elastic transformations offer the advantage to handle local distortions. Within the elastic transformations, two radial basis functions have been used in the reviewed CAD for CaP systems: (i) the Thin Plate Spline (TPS) and (ii) the B-splines. Apart from the formalism, these two approaches have a main difference. With B-splines, the control points are usually uniformly and densely placed on a grid whereas with TPS, the control points correspond to detected or selected key points. For instance, by using TPS, Mitra et al. in [155] obtained more accurate and time efficient results than with the B-splines strategy [156].

In general only rigid or affine registrations have been used to register multi-parametric images from a same protocol whereas elastic registration methods are more commonly used to register multi-protocol images (e.g., histopathology with MRI images) [146, 157].

\subsubsection{Similarity measure}

The most naive similarity measure used in the reviewed registration frameworks is the Mean Squared Error (MSE) of the SI of MRI images. However, this metric is not well suited when multi-parametric images are involved due to the tissue appearance variations between the different modalities. In that regard, Mutual Information (MI) was introduced as a registration measure in the late 1990's in [158]. The MI measure finds its foundation in the assumption that a homogeneous region in the first modality image should also appear as a homogeneous region in the second modality even if their SIs are not identical. Thus, those regions share information and the registration task can be achieved by maximizing this common information. Maximizing the $\mathrm{MI}$ is in fact equivalent to minimizing the joint entropy which is a measure related with the degree of uncertainty or dispersion of the data in the joint histogram. A generalized form of MI, Combined Mutual Information (CMI), was proposed in [159]. CMI encompasses interdependent information such as texture and gradient into the metric.

\subsubsection{Optimization methods}

Registration is usually regarded as an optimization problem where the parameters of the geometric transformation model have to be inferred by minimizing the similarity measure. Iterative estimation methods are commonly used being the L-BFGS-B quasi-Newton method [160] and gradient descent [161] the most common ones. During our review, we noticed that authors do not usually linger over optimizer choice. 


\subsubsection{Interpolation}

The registration procedure involves transforming an image, and pixels mapped to non-integer points must be approximated using interpolation methods. As for the optimization methods, we notice that little attention has been paid on the choice of those interpolations methods. However, commonly used methods are bilinear, nearest-neighbour, bi-cubic, spline and inverse-distance weighting method [162].

\subsubsection{Registration methods used in CAD system}

Studies presenting a CAD pipeline incorporating an automatic registration procedure are summarized in Table 3. Ampeliotis et al. in $[101,102]$ did not use a general registration framework to register 2D $\mathrm{T}_{2}-\mathrm{W}$ and DCE images. By using image symmetries and the MSE metric, they found the parameters of an affine transformation but without using a common objective function. They were finding independently and sequentially the scale factor, the rotation and finally the translation.

Giannini et al. [163] used also a in-house registration for $2 \mathrm{D} \mathrm{T}_{2}-\mathrm{W}$ and $\mathrm{DW}$ images using an affine model. The bladder is first segmented in both modalities in order to obtain its contours and to focus the registration. Giannini et al. [163] and also Vos et al. [164] used the same framework which is based on finding an affine transformation to register the $\mathrm{T}_{2}-\mathrm{W}$ and DCE images using MI [165]. Then, an elastic registration using B-spline takes place using the affine parameters to initialize the geometric model with the same similarity measure. However, the approaches differ regarding the choice of the optimizer since a gradient descent is used in [163] and the same optimization problem is tackled via a quasi-Newton method in [164]. Moreover, Giannini et al. [163] performed a 2D registration whereas Vos et al. [164] registered 3D volumes.

Viswanath et al. in [145, 110] as well as Vos et al. [137] performed an affine registration using the MI as similarity measure to correct the misalignment between $\mathrm{T}_{2}-\mathrm{W}$ and DCE images. The choice of the optimizer was not specified. Viswanath et al. [145, 110] focused on 2D registration while Vos et al. [137] performed $3 \mathrm{D}$ registration.

Finally, Viswanath et al. in [118] performed a 3D registration with the three modalities, $\mathrm{T}_{2}-\mathrm{W}$ and DCE and DW MRI, by using an affine transformation model combined with the CMI similarity measure as presented in [159]. Moreover, in this latter work, the authors employed a gradient descent approach to solve this problem but suggested Nelder-Mead simplex and quasi-Newton method as other solutions.

\section{CADe - CADx}

\subsection{CADe: ROIs detection/selection}

As discussed in the introduction and shown in Fig. 1, the image classification framework is often composed of a CADe and a CADx. In this section, we will focus on studies embedding a CADe in their framework. Two approaches are considered to define a CADe (see Table 6): (i) voxel-based delineation and (ii) lesion 
segmentation. The first strategy, which concerns the majority of the studies reviewed (see Table 6), is in fact linked to the nature of the classification framework $[114,115,163,128,166,104,135,167,98,99,126,168$, $169,170,151,171,172,132,173,174,145,110,118,119]$. All voxels are considered as a possible lesion and the output of the framework will be pixels classified as lesion and non lesion. The second group of methods is composed of method implementing a lesion segmentation algorithm to delineate potential candidates to further obtain a diagnosis through the CADx. This approach was borrowed from other application areas such as breast cancer. These methods are in fact very similar to the classification framework used in CADx later.

Vos et al. [149] highlighted lesion candidates by detecting blobs in the ADC map. These candidates were filtered using some a priori criteria such as SI or diameter. The candidate blobs detected are then filtered depending on their appearances (cf. maximum of the likelihood of the region, diameter of the lesion) and their SI in ADC and $\mathrm{T}_{2}-\mathrm{W}$ images. The detected regions are then used as inputs for the CADx.

Litjens et al. [148] used a pattern recognition approach in order to delineate the ROIs. A blobness map was calculated in the same manner as previously in [164] using the multi-resolution Hessian blob detector on the ADC map, $\mathrm{T}_{2}-\mathrm{W}$ and pharmacokinetic parameters maps (see Sect. 4.2 for details about those parameters). Additionally, the position of the voxel $\mathbf{x}=\{x, y, z\}$ was used as a feature as well as the Euclidean distance of the voxel to the prostate center. Hence, the feature vectors were composed of eight features and a Support Vector Machines (SVM) classifier was trained using a Radial Basis Function (RBF) kernel (see Sect. 4.4 for more details).

Subsequently, Litjens et al. in [139] modified this approach by including only features related to the blob detection on the different maps as well as the original SIs of the parametric images. Two new maps were introduced based on texture. Instead of a SVM classifier, a $k$-Neareast Neighbour $(k$-NN) classifier was used. The candidate regions were then extracted by performing a local maxima detection followed by post-processing region-growing and morphological operations.

In a similar way, Litjens et al. in [142] used the same approach and added two new features: (i) a Gaussian texture bank on $\mathrm{T}_{2}$-W to create new maps and (ii) the DW MRI image acquired with $b=800$ s.mm ${ }^{-}$2. Three classifiers were tested: Linear Discriminant Analysis (LDA), GentleBoost and Random forest. After evaluation, random forest was selected as classifier due to its overall performances.

\subsection{CADx: Feature detection}

Discriminative features which can be used to recognize CaP from healthy tissue have to be first detected. This processing is known in computer vision as feature extraction. However, feature extraction is also the name given in pattern recognition to some types of dimension reduction methods which will be presented in the next section. In order to avoid confusion between these two aspects, in this survey, the procedure "detecting" or "extracting" features from images and signals will be defined as feature detection. This 
section will summarize the different strategies employed for this task. The features used in the studies reviewed are summarized in Table 7.

\subsubsection{Image-based features}

This section will focus on image-based features detection. Two main strategies to detect features have been identified and used for the purpose of our classification: (i) voxel-wise detection and (ii) region-wise detection.

- Voxel-wise features: This strategy refers to the fact that a feature is extracted at each voxel location. $\mathrm{CaP}$ as previously discussed (see Table 1) can be discerned due to SI changes. Hence, intensity-based features are one of the most common features used to build the feature vector which has to be classified $[101,102,114,115,13,175,148,139,142,166,121,122,145,118]$. This type of feature consists simply of the SI of each voxel of the different MRI modalities.

Edge based features have also been used to detect SI changes. Each feature is computed by convolving the original image with an edge operator. Three of these operators are used: (i) Prewitt operator [176], (ii) Sobel operator [177] and (iii) Kirsch operator [178]. Results obtained with these operators vary, due to their different kernels. These features are commonly incorporated in the feature vector for further classification in the CAD systems reviewed [121, 122, 171, 172, 173, 174, 118].

Gabor filters $[179,180]$ offer another approach to extract information related to edges and texture and were integrated in three different CAD for $\mathrm{CaP}[174,119,132]$.

Texture-based features provide other characteristics discerning CaP from healthy tissue. The most common texture analysis for image classification are co-occurrence matrices with their related statistics which were proposed in [181] and are commonly used in CAD systems [182, 121, 122, 171, 172, 173, $174,145,118,119]$. Fractal analysis and more precisely a local estimation of the fractal dimension [183] describing the texture roughness at a specific location was used in [104]. A wavelet-based method in a multi-resolution framework was used to estimate the fractal dimension. Cancerous tissue showed a higher fractal dimension than healthy tissue.

Chan et al. [13] described the texture using the frequency signature via the Discrete Cosine Transform (DCT) [184] defining a neighbourhood of $7 \times 7$ pixels for each of the modalities that they used. Similarly, Viswanath et al. in [119] projected $\mathrm{T}_{2}-\mathrm{W}$ images into the wavelet space and used the coefficients obtained from the decomposition as features. The wavelet family used for the decomposition was the Haar wavelet. Finally, Litjens et al. in [142] computed texture map based on $\mathrm{T}_{2}$-W images using a Gaussian filter bank [185].

The position of a voxel within the prostate was also considered a feature. Authors in [148, 142] computed the Euclidean distance from each voxel to the prostate center as well as the individual distance 
in the three directions $x, y$ and $z$. Chan et al. [13] embedded the same information but using cylindrical coordinates $r, \theta$ and $z$ instead, corresponding to the radius, azimuth and elevation respectively.

- Region-wise features: Unlike the previous section, another strategy is to study an entire region and extract characteristic features corresponding to this region. The most common approach reviewed can be classified as statistical methods. First, a feature map is computed for the whole image instead of using single voxels. Then, ROIs are defined and statistics are extracted from each of these regions. The most widely used statistic is based on percentiles $[182,148,139,142,82,171,172,173,174,145,118$, $119,137,138,164,149]$. The percentile used is usually manually determined observing the distribution and corresponds to the best discriminant value differentiating malignant and healthy tissue. In addition, statistic-moments such as mean, standard deviation, kurtosis and skewness are also used [101, 102, 182, 121, 122, 82]. Litjens et al. in [142] also introduced a feature based on symmetry. They compute the mean of a candidate lesion as well as its mirrored counter-part and compute the quotient as feature.

Another subset of features are anatomic which were also used in [139, 142, 135]. Litjens et al. in $[139,142]$ computed the volume, compactness and sphericity related to the region to integrate it in their feature vector. Matulewicz et al. [135] introduced four features corresponding to the percentage of tissue belonging to the regions $\mathrm{PZ}, \mathrm{CG}$, periurethral region or outside prostate region for the considered ROI.

In contrast to anatomical are histogram-based feature descriptors. For instance, Liu et al. [116] introduced four different types of histogram-based features. The first type corresponds to the histogram of the SI of the image. The second type is the Histogram of Oriented Gradient (HOG) [186]. HOG descriptor describes the local shape of the object of interest by using the distribution of gradient directions. The third histogram-based type used in [116] was shape context [187]. The shape context is also a way to describe the shape of an object of interest. The last set of histogram-based feature extracted is based on the framework described in [188] which is using the Fourier transform of the histogram created via Local Binary Pattern (LBP) [189].

The last group of region-based feature is based on fractal analysis. The features proposed are based on estimating the fractal dimension which is a statistical index representing the complexity of what is analysed. Lv et al. [111] proposed two features based on fractal dimension: (i) texture fractal dimension and (ii) histogram fractal dimension. Lopes et al. [104] proposed a 3D version to estimate the fractal dimension of a volume using wavelet decomposition.

\subsubsection{DCE-based features}

DCE-MRI is more commonly based on a SI analysis over time as presented in Sect. 2.2.2. The features extracted for DCE-MRI analysis are presented. 
- Whole-spectra approach: Some studies are using the whole DCE time series as feature vector [101, 102, $132,145,174]$. In some cases, the high-dimensional feature space is reduced using dimension reduction methods as it will be presented in the next section (see Sect.4.3).

- Semi-quantitative approach: Semi-quantitative approaches are based on mathematically modelling the DCE time series. The parameters modelling the signal are commonly used mainly due to the simplicity of their computation. Parameters included in semi-quantitative analysis are summarized in Table 8 and also graphically depicted in Fig.7. A set of time features corresponding to specific amplitude level (start, maximum and end) are extracted. Then, derivative and integral features are also considered as discriminative and are commonly computed.

- Quantitative approach: As presented in Sect. 2.2.2, quantitative approaches correspond to mathematicalpharmacokinetic models based on physiological exchanges. Four different models have been used in CAD for $\mathrm{CaP}$ systems. The most common model reviewed was the Brix model using three parameters $A, k_{e p}$ and $k_{e l}[114,115,168,166,98,99]$. The Tofts model [190] and more precisely the parameters $K_{\text {trans }}, k_{e p}$ and $v_{e}$ were used in $[175,148,139,142,163,121,122,167]$.

Mazzetti et al. [167] and Giannini et al. [163] used the Weibull function in a different empirical model based on the West-like function and referred to as the phenomenological universalities model [191] defined by three parameters $\beta, a_{0}$ and $r$. For all these models, the parameters are inferred using an optimization curve fitting approach.

\subsubsection{MRSI-based features}

- Whole spectra approach: As in the case of DCE analysis, one common approach is to incorporate the whole MRSI spectra in the feature vector for classification $[128,126,169,151,173,171,172,145,135]$. Sometimes post-processing involving dimension reduction methods is performed to reduce the complexity during the classification as it will be presented in Sect. 4.3.

- Quantification approach: We can reiterate that in MRSI only few biological markers (cf., choline, creatine and citrate metabolites mainly) are known to be useful to discriminate CaP and healthy tissue. Then, concentrations of these metabolites can be considered as a feature used for classification. In order to perform this quantification, four different approaches have been used. The QUEST [192], AMARES [193] and VARPRO [194] models were used in [128]. They are all time-domain quantification methods varying by the type of pre-knowledge embedded and the optimization approaches used to solve the quantification problem. Unlike the time-domain quantification approaches, Parfait et al. [126] used the LcModel approach [195] which solves the optimization problem in the frequency domain.

Although Parfait et al. [126] used each metabolite concentrations individually, other authors such as 
Kelm et al. [128] proposed to compute relative concentrations as the ratio of choline plus creatine to citrate or the ratio of citrate to choline plus creatine plus citrate.

- Wavelet decomposition approach: Tiwari et al. [132] performed a wavelet packet decomposition [196] of the spectra with the Haar wavelet basis function and used its coefficients as features.

\subsection{CADx: Feature selection and feature extraction}

As presented in the previous section, a wide variety of features can be computed (see Table 7). This often leads from multi-parametric MRI data to a high dimensions feature space which might mislead or corrupt the classifier used for training. Thus, it is often of interest to reduce the number of dimensions before proceeding to the classification task. The strategies used can be grouped as: (i) feature selection and (ii) feature extraction and those methods will be presented in the above sections. However, only the methods used in CAD system are presented and summarized in Table 9.

\subsubsection{Feature selection}

The feature selection strategy is based on selecting the most discriminative feature dimensions of the high-dimensional space. Thus, the low-dimensional space is then composed of a subset of the original features detected. In this section, methods employed in the studies reviewed will be briefly presented. More extensive reviews specific to feature selection can be found in [197]. Niaf et al. [121, 122] make use of the p-value by using the independent two-sample t-test with equal mean for each feature dimension. The features can be ranked and the most significant features can be selected. However, this technique suffers from a main drawback since it assumes that each feature is independent, which is unlikely to happen and introduces a high degree of redundancy in the features selected.

Vos et al. in [149] employed a similar feature ranking approach but make use of the Fisher discriminant ratio to compute the relevance of each feature dimension. Once the features are ordered, the authors select the feature dimensions with the larger Fisher discriminant ratio.

MI can also be used to select a subset of feature dimensions. Peng et al. introduced two main criteria to select the feature dimensions: (i) maximal relevance and (ii) minimum redundancy. Combination of these two criteria is known as minimum Redundancy Maximum Relevance (mRMR) ${ }^{2}$ [198] and are computed as a difference or quotient. Authors in $[121,122]$ make use of maximal relevance criterion alone and also of both mRMR difference and quotient criterion. Viswanath et al. in [119] also reduced their feature vector via mRMR difference and quotient.

\footnotetext{
${ }^{2}$ mRMR implementation can be found at: http://penglab.janelia.org/proj/mRMR/
} 


\subsubsection{Feature extraction}

The feature extraction strategy is related to dimension reduction methods but not selecting discriminative features. Instead, these methods aim at mapping the data from the high-dimensional space into a lowdimensional space created to maximize the separability between the classes. The mapping can be performed in a linear or a non-linear manner. Again, only methods employed in $\mathrm{CAD}$ for $\mathrm{CaP}$ system will be reviewed in this section. We refer the reader to [199] for a full review of feature extraction techniques.

Principal Components Aanalysis (PCA) is the most commonly used linear mapping method in CAD prostate [200]. Tiwari et al. in [170, 151, 132] used PCA in order to reduce the dimensionality of their feature vector. Non-linear mapping was also used for dimension reduction. It is mainly based on Laplacian eigenmaps and Locally Linear Embedding (LLE) methods. Laplacian eigenmaps ${ }^{3}$, also referred as spectral clustering in computer vision, aim to find a low-dimensional space in which the proximity of the data should be preserved from the high-dimensional space $[152,201]$. Tiwari et al. in $[169,151,171]$ and Viswanath et al. in [174] used this spectral clustering to project their feature vector into a low-dimensional space. The feature space in these studies is usually composed of features extracted from a single or multiple modalities and then concatenated before applying the Laplacian eigenmaps dimension reduction technique. Tiwari et al. in $[151,173]$ used a slightly different approach by combining the Laplacian eigenmaps techniques with a prior multi-kernel learning strategy.

$\mathrm{LLE}^{4}$ is another common non-linear dimension reduction technique widely used, first proposed in [202]. Tiwari et al. in [170] used a modified version of the LLE algorithm in which they applied LLE in a bagging approach with multiple neighbourhood sizes. The different embeddings obtained are then fused using the Maximum Likelihood (ML) estimation.

\subsection{CADx: Classification}

\subsubsection{Classifier}

Once the feature vector has been extracted and eventually the complexity reduced, it is possible to make a decision and classify this feature vector to belong to $\mathrm{CaP}$ or healthy tissue. Classification methods used in a $\mathrm{CAD}$ for $\mathrm{CaP}$ system to distinguish these two classes are summarized in Table 10. A full review of classification methods used in pattern recognition can be found in [203].

- Rule-based methods: Lv et al. [111] make use of a decision stump classifier to distinguish CaP and healthy classes. Puech et al. [136] detect CaP by implementing a given set of rules using a score scheme in a medical decision making approach. The feature values are compared with a pre-defined threshold.

\footnotetext{
${ }^{3}$ Laplacian eigenmap implementation is available at: http://www.cse.ohio-state.edu/ mbelkin/algorithms/algorithms. html

${ }^{4}$ LLE implementation is available at: https://www.cs.nyu.edu/ roweis/lle/
} 
Then, at each comparison, the final score is incremented, depending on the threshold and the final decision is taken depending of this final score.

- Clustering methods: $k$-Neareast Neighbour $(k-\mathrm{NN})$ is one of the simplest supervised machine learning classification methods. $k$-NN was one of the methods used in $[121,122]$ mainly to make a comparison with different machine learning techniques. Litjens et al. in [139] used this method to roughly detect potential $\mathrm{CaP}$ voxels before performing a region-based classification.

The $k$-means algorithm is an unsupervised clustering method in which the data is iteratively partitioned into $k$ clusters. This algorithm can also be used for "on-line" learning. In case that new data has to be incorporated, the initial centroid positions correspond to the results of a previous $k$-means training and is followed by the assignment-updating stage previously explained. Tiwari et al. in [169, 151] used $k$-means with three clusters corresponding to $\mathrm{CaP}$, healthy and non-prostate, respectively. $k$-means was applied iteratively and the voxels corresponding to the largest cluster were excluded under the assumption that it was assigned to "non-prostate" cluster. The algorithm stopped when the number of voxels in all remaining clusters was smaller than a given threshold. Tiwari et al. in [170] and Viswanath et al. in $[174,145]$ used $k$-means in a repetitive manner in order to be less sensitive to the centroids initialisation. Thus, $k$ clusters were generated $T$ times. The final assignment was performed by majority voting using a co-association matrix as proposed in [204].

- Linear model classifiers: Linear Discriminant Analysis (LDA) can be used as a classification method in which the optimal linear separation between two classes is found by maximizing the interclass variance and minimizing the intraclass variance [205]. LDA has been used in [182, 13, 142, 121, 122, 149]. Logistic regression can be used to perform binary classification and can provide the probability of an observation to belong to a class. This has been used to create a linear probabilistic model in [128, 136].

- Non-linear model classifier: Viswanath et al. in [119] used Quadratic Discriminant Analysis (QDA) instead of LDA. Unlike in LDA in which one assumes that the class covariance matrix $\Sigma$ is identical for all the classes, in QDA, a covariance matrix $\Sigma_{k}$ specific to each class is computed.

- Probabilistic classifier: The most commonly used classifier is the naive Bayes classifier which is a probabilistic classifier assuming independence between each feature dimension [206]. The Naive Bayes classifier has been used in $[163,167,121,122]$. The Normal distribution was adopted as the likelihood probability for that model.

- Ensemble learning classifiers: AdaBoost is an adaptive method based on an ensemble learning method and was initially proposed by [207]. AdaBoost linearly combines several weak learners resulting into a final strong classifier. A weak learner is defined as a classification method performing slightly 
better than random classification. Random forest ${ }^{5}$ is a classification method which is based on creating an ensemble of decision trees and was introduced in [208]. Probabilistic boosting-tree is another ensemble learning classifier which shares principles with AdaBoost but using them inside a decision tree [209]. Lopes et al. [104] make use of the AdaBoost classifier to perform their classification while Litjens et al. in [142] used the GentleBoost variant [210] which provides a modification of the function affecting the weight at each weak classifier. The random forest classifier has been used in [128, 142, 132, 173, 110] whereas the probabilistic boosting-tree classifier in [171, 132, 172, 118].

- Kernel method: A Gaussian process ${ }^{6}$ for classification is a kernel method in which it is assumed that the data can be represented by a single sample from a multivariate Gaussian distribution [211]. Only the work of Kelm et al. [128] used a Gaussian process for classification in MRSI data.

- Sparse kernel methods: In a classification scheme using Gaussian processes, when a prediction has to be performed, the whole training data will be used to assign a label to the new observations. That is why this method is also called kernel method. Sparse kernel category is composed of methods which rely only on a few labelled observations of the training set to assign the label of new observations [203].

Support Vector Machines $(\mathrm{SVM})^{7}$ is a sparse kernel method which aims at finding the best linear hyperplane (non-linear separation is discussed further) which separates two classes such that the margin between the two classes is maximized [212]. SVM can also be used as a non-linear classifier by performing a kernel trick [213]. The original data $\mathbf{x}$ can be projected to a higher-dimension space in which it is assumed that a linear hyperplane will better split the classes. Different kernels are popular such as the RBF kernel, polynomial kernels or Gaussian kernel. In prostate CAD system, SVM is the most popular classification method and was used in a multitude of research works $[114,115,13,128,148,139,116,104,121,122$, $98,99,126,82,168,132,137,138,164,149]$.

Relevant Vector Machine (RVM) is a sparse version of Gaussian process previously presented and was proposed by [214]. RVM is identical to a Gaussian process with a specific covariance function [215]. Ozer et al. [98, 99] make use of RVM and make a comparison with SVM for the task of CaP detection.

- Neural network: Multilayer perceptron is a feed-forward neural networks considered as the most successful model of this kind in pattern recognition [203]. The most well known model used is based on two layers. Matulewicz et al. [135] as well as Parfait et al. [126] used this classifier to classify MRSI spectra. Probabilistic neural networks are another type of feed-forward networks which can be derived from the multilayer perceptron case and was proposed by [216]. This classifier can be modelled by

\footnotetext{
${ }^{5}$ Random forest implementation can be found at: http://www.stat.berkeley.edu/ breiman/RandomForests/cc_software. htm

${ }^{6}$ Gaussian process implementation can be found at: http://www.gaussianprocess.org/gpml/code/matlab/doc/index.html ${ }^{7} \mathrm{SVM}$ implementation can be found at: http://www.csie.ntu.edu.tw/ cjlin/libsvm/
} 
changing the activation function of the hidden layer to an exponential function. This method was used in $[101,102,118]$.

- Graphical model classifiers: Markov Random Field (MRF) can also be used for classification in order to perform a lesion segmentation method to detect CaP. Liu et al. [166] and Ozer et al. [99] used MRF as an unsupervised method to segment lesions in multi-parametric MRI. Artan et al. [114, 115] used conditional random fields instead of MRF for MRI segmentation.

\subsubsection{Model validation}

In pattern recognition, the validation model for assessing the performance of a classifier plays an important role in the final results. Two techniques are broadly used in the development of a CAD system and are summarized in Table 11. The most popular technique (see Table 11) is the Leave-One-Out Cross-Validation (LOOCV) technique. From the whole data, one patient is kept for validation and the other cases are used for training. This manipulation is repeated until each patient has been used for validation. This technique is popular when working with a limited number of patients, allowing to train on a representative number of cases even with a small dataset. However, Leave-One-Out Cross-Validation (LOOCV) can suffer from large variance and can be considered as an unreliable estimate [217].

The other technique is the $k$-fold Cross-Validation ( $k$-CV) technique which is based on splitting the dataset into $k$ subsets where the samples are randomly selected. Then, one fold is kept for the validation and the remaining subsets are used for training. The classification is then repeated as in the LOOCV technique. In fact Leave-One-Out Cross-Validation (LOOCV) is a particular case of $k$-fold Cross-Validation $(k$-CV) when $k$ equals the number of patients. In the reviewed papers, the typical values used for $k$ were set to three and five. $k$-fold Cross-Validation $(k-\mathrm{CV})$ is regarded as more appropriate than Leave-One-Out Cross-Validation (LOOCV), but the number of patients in the dataset needs to be large enough for the results to be meaningful.

\subsubsection{Evaluation measure}

Several metrics can be used in order to assess the performance of a classifier and are summarized in Table 12. Voxels in the MRI image are classified into healthy or malign tissue and compared with a groundtruth. This allows to compute a confusion matrix by counting true positive, true negative, false positive and false negative samples. From this analysis, different statistics can be extracted.

The first statistic used is the accuracy which is computed as the ratio of true detection to the number of samples. However, depending on the strategy employed in the CAD work-flow, this statistic can be highly biased by a high number of true negative samples which will boost the accuracy score overestimating the actual performance of the classifier. 
That is why, the most common statistic computed are sensitivity and specificity which give a full overview of the performance of the classifier. Sensitivity is also called the true positive rate and is equal to the ratio of the true positive samples over the true positive added with the false negative samples as shown in Eq. (5). Specificity is also named the true negative rate and is equal to the ratio of the true negative samples over the true negative added with the false positive samples as shown in Eq. (6).

$$
\begin{aligned}
& S E N=\frac{T P}{T P+F N}, \\
& S P E=\frac{T N}{T N+F P} .
\end{aligned}
$$

These statistics can be used to compute the Receiver Operating Characteristic (ROC) curves [218]. This analysis represents graphically the sensitivity as a function of (1 - specificity), which is in fact the false positive rate, by varying the discriminative threshold of the classifier. By varying this threshold, more true negative samples will be found but often at the cost of detecting more false negatives. However, this fact is interesting in CAD since it is possible to obtain a high sensitivity and to ensure that no cancers are missed even if more false alarms have to be investigated. A statistic derived from ROC analysis is the Area Under the Curve (AUC) which corresponds to the area under the ROC and is a measure used to make comparisons between models.

The ROC analysis can be classified as a pixel-based evaluation method. However, a cancer can be also considered as a region. The Free-Response Receiver Operating Characteristic (FROC) extends the ROC analysis but to a region-based level. The same confusion matrix can be computed were the sample are not pixels but lesions. However, it is important to define what is a true positive sample in that case. Usually, a lesion is considered as a true positive sample if the region detected by the classifier overlaps "sufficiently" the one delineated in the ground-truth. However, "Sufficiently" is a subjective measure defined by each researcher and can correspond to one pixel only. However, an overlap of 30 to $50 \%$ is usually adopted. Finally, in addition to the overlap measure, the Dice's coefficient is often computed to evaluate the accuracy of the lesion localization. This coefficient consists of the ratio between twice the number of pixels in common and the sum of the pixels of the lesions in the ground-truth $G T$ and the output of the classifier $S$, defined as shown in Eq. (7).

$$
Q_{D}=\frac{2|G T \cap S|}{|G T|+|S|}
$$




\section{Discussion}

\subsection{Results reported}

As discussed previously in Sect.4.4.3, different metrics have been used to report results. A comparison of the different methods reviewed is given depending on the metric used in field of research and also the type of MRI scanner used (cf., 1.5 versus 3.0 Tesla). For each field, the best performances obtained in each study were reported in these figures. The results given in terms of AUC-ROC are depicted in Fig. 9. The results vary between $71 \%$ and $97 \%$ for some experiments with a 1.5 Tesla MRI scanner and $77 \%$ and $95 \%$ with a 3.0 Tesla MRI scanner.

The results in regard of sensitivity and specificity are reported in Fig. 10. In the case that the data were collected with a 1.5 Tesla MRI scanner, the sensitivity ranges from $74 \%$ to $100 \%$ and the specificity from $43 \%$ to $93 \%$. For the experiments carried out with a 3.0 Tesla MRI scanner, the sensitivity varies from $60 \%$ to $90 \%$ and the specificity from $66 \%$ to $99 \%$. Four studies also use FROC analysis to report their results and are reported in Fig. 8.

\subsection{Comparison}

We would like to stress the following findings drawn during the review of the different studies:

1. Quantitatively, it is difficult to make a fair comparison between the different studies reviewed. Different factors come into play to elucidate this fact. Mainly a lack of standardization can be pointed out in regard to experimental evaluation: (i) different datasets are used during the evaluation of the frameworks developed hinderng a inter-study comparison. The same conclusion has been recently drawn by [142] supporting this argument; (ii) the experimental results are not reported with a common metric which leads to the inability to compare the different studies.

2. However, multiple studies reported some performance improvements using multi-parametric imaging techniques instead of mono-parametric imaging techniques. Considering only the most recent studies proposing CADe-CADx frameworks, the following results can be highlighted. Viswanath et al. [118] obtained an AUC of $77 \%$ using an ensemble learning approach combining the features from the three modalities $\mathrm{T}_{2}$-W-DCE-DW MRI, while the results obtained as standalone modality were ranging from $62 \%$ to $65 \%$. Tiwari et al. [173] drawn similar conclusions by using $\mathrm{T}_{2}-\mathrm{W}$ and MRSI modalities as both in standalone and multi-parametric frameworks with an improved AUC ranging from 57\%-76\% to $85 \%$. The most recent work of Litjens et al. [142] obtained an improved AUC metric from 71\%-76\% considering each modality separately (e.g., $\mathrm{T}_{2}$-W-DCE-DW MRI) to $89 \%$ in their multi-parametric framework. 
3. The studies comparing particular combination of more than one modality give rise to the same fact [99, $148,116,142]$ : using three modalities lead to better performances than using any combination of two modalities.

4. Unlike the previous remark 2, no straightforward conclusions can be given regarding the performances of each modality in a standalone framework. The modality being processed by different methods, it does not allow us to conclude if a modality by itself is more suited than another. However, we were able to distinguish some interesting trends which deserves the attention of the community. Tiwari et al. in $[171,132,173]$ observed that MRSI is a more suitable modality than $\mathrm{T}_{2}$-W to highlight cancers. Moreover, ADC maps have shown a better discriminative power than $\mathrm{T}_{2}$-W as well $[175,118,82]$. Lately, Litjens et al. in [142] observed that DW modality was more suitable than both DCE and $\mathrm{T}_{2}$-W to distinguish CaP in their CADx system.

5. Furthermore, multi-parametric has attracted the attention of both radiologists and computer vision researchers. Indeed, pioneer research groups included new modalities over years when at the same time, new research groups directly introduced multi-parametric CAD systems. These facts lead us to think that $\mathrm{CaP}$ researches will benefit from multi-parametric imaging techniques.

6. When focusing on the different modalities used, it can be pointed out that no research reported the use of all modalities in a single framework: MRSI is usually used as a standalone modality and never combined with the three remaining. Nevertheless, this modality has shown some overall good performances at the price of a lower resolution as well as an increased acquisition time. Moreover, MRSI analysis is more complex in comparison with the other modalities. To our mind, MRSI could contribute in a multi-parametric framework and should be fused with the other modalities.

7. Lately, three studies focused on developing a region-based classification in which PZ and CG will be analysed separately $[119,139,142]$. The promising results were obtained which indicates that this strategy should be further investigated.

8. Recent studies are using quantitative features in addition to SI. It seems that these quantitative features provide uncorrelated information with respect to SI features and should lead to better performances when combined all together.

9. Regarding the methods used in the "image regularisation" (cf., pre-processing, segmentation and registration), it is particularly difficult to distinguish the benefit of a method over another since none of the studies focus on making comparison of these processing stages. The focus is usually entirely based on the "image classification" framework where different methods are directly compared. Note 
that the performance of a classifier is highly linked with the features vector extracted from particular data. Hence, one can not conclude that a machine learning method is more appropriate than another, but we can identify a trend in which SVM as well as ensemble learning classifiers (e.g., AdaBoost, GentleBoost and random forest) seem to perform better than neural network, LDA or Naive Bayes.

10. We would like to draw the attention of the reader on the feature extraction/selection stage. This processing could reduce the complexity and also find a better feature space for classification. However, few studies are performing such approaches. Niaf et al. [121, 122] are successfully applying a scheme to reduce the number of dimensions by selecting the most discriminative features. It allows them to obtain improved performances compared with a classification performed with their initial feature vector. Another group of studies also applied different feature extraction methods [145, 174, 119, 169, $170,151,172,132,173]$. In these specific cases, no comparison is performed against the original data.

\subsection{General discussion}

This review leads to some general discussions which could direct to future avenues for research. As previously mentioned, no open multi-parametric dataset is currently available. This fact leads to an impossibility to fairly compare the different algorithms designed over years. Also, the availability of a full multi-parametric MRI dataset, could lead to the development of algorithms which use all the different modalities currently available. Recalling Table 2, it can be noted that none of the current works provides a solution using at the same time the four different modalities. Also, all the algorithms are focused on one type of scanner only, either 1.5 Tesla and 3.0 Tesla. A dataset including both these types of imaging could allow development of more generic algorithms.

Analysing the different stages of the CAD work-flow, it is seen that the current CAD systems do not include all the pre-processing steps. It could be interesting to evaluate the improvement using these preprocessing steps on the final results. Regarding segmentation and registration of the prostate, CAD systems could greatly benefit from specific research in these areas which could lead to a better automation of those systems. Moreover, other segmentation and registration methods not currently used in CAD systems could also obtain better results.

Regarding the classification framework, it seems that the current well-known pattern recognition methods have been widely studied. However, more investigations should be carried out regarding the feature detection stage. Lately, histogram-based features have shown good capabilities in the field of computer vision and could be further investigated. Only one study by [116] used some of these features.

An important point allowing a fair comparison between methods resides in the fact that no common dataset, nor universal evaluation model, nor metric has been defined by the research community allowing such comparison. This review aims to have an impact in that respect by providing a novel publicly available multi- 
parametric and multi-vendor MRI dataset (from a 1.5 Tesla General Electric scanner and a 3.0 Tesla Siemens scanner). This dataset is available at the following website address: http://visor.udg.edu/dataset. The dataset is composed of the four modalities discussed in this review with their corresponding ground-truth images. For each scanner type, each subset is composed of twenty patients with cancerous lesions and ten healthy patients, having a total of 60 patients. In addition of the repository activity, this website will aim at providing comparison between algorithms developed by the research community.

\section{Conclusion}

This review has presented an overview and classification of the research related to CAD development for CaP using multi-parametric MRI data. We aimed at providing background information regarding multiparametric MRI imaging techniques and a description of the work-flow in the different CAD stages. The methods used in the literature for each of these stages have been reviewed along with the available results of the CAD systems. Moreover, insight discussions and possible future research directions have also been given. Finally, a multi-parametric multi-vendor dataset has been made available to the research community in order to provide a standardised platform for $\mathrm{CAD}$ development and evaluation for $\mathrm{CaP}$ using multi-parametric MRI.

\section{Acknowledgement}

G. Lemaitre was supported by the Generalitat de Catalunya (grant nb. FI-DGR2012) and partly by the Mediterranean Office for Youth (grant nb. 2011/018/06).

We would like to acknowledge Sharad Nagappa for all the discussions involved and his precious advices and corrections regarding the redaction of this entire manuscript.

\section{References}

[1] M. L. Giger, H. P. Chan, J. Boone, Anniversary paper: History and status of CAD and quantitative image analysis: the role of Medical Physics and AAPM, Med Phys 35 (12) (2008) 5799-5820.

[2] T. Hambrock, P. C. Vos, C. A. Hulsbergen-van de Kaa, J. O. Barentsz, H. J. Huisman, Prostate cancer: computer-aided diagnosis with multiparametric 3-T MR imaging-effect on observer performance, Radiology 266 (2) (2013) 521-530.

[3] H. P. Chan, B. Sahiner, M. A. Helvie, N. Petrick, M. A. Roubidoux, T. E. Wilson, D. D. Adler, C. Paramagul, J. S. Newman, S. Sanjay-Gopal, Improvement of radiologists' characterization of mammographic masses by using computeraided diagnosis: an ROC study, Radiology 212 (3) (1999) 817-827.

[4] J. C. Dean, C. C. Ilvento, Improved cancer detection using computer-aided detection with diagnostic and screening mammography: prospective study of 104 cancers, AJR Am J Roentgenol 187 (1) (2006) 20-28.

[5] F. Li, M. Aoyama, J. Shiraishi, H. Abe, Q. Li, K. Suzuki, R. Engelmann, S. Sone, H. Macmahon, K. Doi, Radiologists' performance for differentiating benign from malignant lung nodules on high-resolution CT using computer-estimated likelihood of malignancy, AJR Am J Roentgenol 183 (5) (2004) 1209-1215. 
[6] N. Petrick, M. Haider, R. M. Summers, S. C. Yeshwant, L. Brown, E. M. Iuliano, A. Louie, J. R. Choi, P. J. Pickhardt, CT colonography with computer-aided detection as a second reader: observer performance study, Radiology 246 (1) (2008) 148-156.

[7] J. V. Hegde, R. V. Mulkern, L. P. Panych, F. M. Fennessy, A. Fedorov, S. E. Maier, C. M. Tempany, Multiparametric MRI of prostate cancer: an update on state-of-the-art techniques and their performance in detecting and localizing prostate cancer, J Magn Reson Imaging 37 (5) (2013) 1035-1054.

[8] H. Hricak, R. D. Williams, D. B. Spring, K. L. Moon, M. W. Hedgcock, R. A. Watson, L. E. Crooks, Anatomy and pathology of the male pelvis by magnetic resonance imaging, AJR Am J Roentgenol 141 (6) (1983) 1101-1110.

[9] R. A. Huch Boni, J. A. Boner, U. M. Lutolf, F. Trinkler, D. M. Pestalozzi, G. P. Krestin, Contrast-enhanced endorectal coil MRI in local staging of prostate carcinoma, J Comput Assist Tomogr 19 (2) (1995) 232-237.

[10] J. Kurhanewicz, D. B. Vigneron, H. Hricak, P. Narayan, P. Carroll, S. J. Nelson, Three-dimensional H-1 MR spectroscopic imaging of the in situ human prostate with high (0.24-0.7-cm3) spatial resolution, Radiology 198 (3) (1996) 795-805.

[11] J. Scheidler, R. Petsch, U. Muller-Lisse, A. Heuck, M. Reiser, Echo-planar diffusion-weighted MR imaging of the prostate, in: Proceedings of the 7th Annual Meeting of ISMRM Philadelphia, 1999, p. 1103.

[12] M. G. Swanson, D. B. Vigneron, T. K. Tran, N. Sailasuta, R. E. Hurd, J. Kurhanewicz, Single-voxel oversampled J-resolved spectroscopy of in vivo human prostate tissue, Magn Reson Med 45 (6) (2001) 973-980.

[13] I. Chan, W. Wells, R. V. Mulkern, S. Haker, J. Zhang, K. H. Zou, S. E. Maier, C. M. Tempany, Detection of prostate cancer by integration of line-scan diffusion, T2-mapping and T2-weighted magnetic resonance imaging; a multichannel statistical classifier, Med Phys 30 (9) (2003) 2390-2398.

[14] S. Wang, K. Burtt, B. Turkbey, P. Choyke, R. Summers, Computer Aided-Diagnosis of prostate cancer on multiparametric MRI: a technical review of current research, BioMed Research International 2014.

[15] J. Ferlay, H. R. Shin, F. Bray, D. Forman, C. Mathers, D. M. Parkin, Estimates of worldwide burden of cancer in 2008: GLOBOCAN 2008, Int. J. Cancer 127 (12) (2010) 2893-2917.

[16] R. Siegel, D. Naishadham, A. Jemal, Cancer statistics, 2013, CA Cancer J Clin 63 (1) (2013) 11-30.

[17] A. C. American Cancer Society, Cancer Facts and Figures 2013, http://www.cancer.org/research/cancerfactsfigures, accessed: 2013-08-01 (2013).

[18] A. C. American Cancer Society, Cancer Facts and Figures 2010, http://www.cancer.org/research/cancerfactsfigures, accessed: 2013-08-01 (2010).

URL http://www. cancer.org/research/cancerfactsfigures

[19] E. Giovannucci, Y. Liu, E. A. Platz, M. J. Stampfer, W. C. Willett, Risk factors for prostate cancer incidence and progression in the health professionals follow-up study, Int. J. Cancer 121 (7) (2007) 1571-1578.

[20] G. D. Steinberg, B. S. Carter, T. H. Beaty, B. Childs, P. C. Walsh, Family history and the risk of prostate cancer, Prostate 17 (4) (1990) 337-347.

[21] M. L. Freedman, C. A. Haiman, N. Patterson, G. J. McDonald, A. Tandon, A. Waliszewska, K. Penney, R. G. Steen, K. Ardlie, E. M. John, I. Oakley-Girvan, A. S. Whittemore, K. A. Cooney, S. A. Ingles, D. Altshuler, B. E. Henderson, D. Reich, Admixture mapping identifies 8q24 as a prostate cancer risk locus in African-American men, Proc. Natl. Acad. Sci. U.S.A. 103 (38) (2006) 14068-14073.

[22] L. T. Amundadottir, P. Sulem, J. Gudmundsson, A. Helgason, A. Baker, B. A. Agnarsson, A. Sigurdsson, K. R. Benediktsdottir, J. B. Cazier, J. Sainz, M. Jakobsdottir, J. Kostic, D. N. Magnusdottir, S. Ghosh, K. Agnarsson, B. Birgisdottir, L. Le Roux, A. Olafsdottir, T. Blondal, M. Andresdottir, O. S. Gretarsdottir, J. T. Bergthorsson, D. Gudbjartsson, A. Gylfason, G. Thorleifsson, A. Manolescu, K. Kristjansson, G. Geirsson, H. Isaksson, J. Douglas, J. E. Johansson, K. Balter, F. Wiklund, J. E. Montie, X. Yu, B. K. Suarez, C. Ober, K. A. Cooney, H. Gronberg, W. J. Catalona, G. V. Einarsson, R. B. Barkardottir, J. R. Gulcher, A. Kong, U. Thorsteinsdottir, K. Stefansson, A common variant associated 
with prostate cancer in European and African populations, Nat. Genet. 38 (6) (2006) 652-658.

[23] I. Agalliu, R. Gern, S. Leanza, R. D. Burk, Associations of high-grade prostate cancer with BRCA1 and BRCA2 founder mutations, Clin. Cancer Res. 15 (3) (2009) 1112-1120.

[24] R. M. Hoffman, F. D. Gilliland, J. W. Eley, L. C. Harlan, R. A. Stephenson, J. L. Stanford, P. C. Albertson, A. S. Hamilton, W. C. Hunt, A. L. Potosky, Racial and ethnic differences in advanced-stage prostate cancer: the Prostate Cancer Outcomes Study, J. Natl. Cancer Inst. 93 (5) (2001) 388-395.

[25] R. W. Ma, K. Chapman, A systematic review of the effect of diet in prostate cancer prevention and treatment, J Hum Nutr Diet 22 (3) (2009) 187-199.

[26] D. D. Alexander, P. J. Mink, C. A. Cushing, B. Sceurman, A review and meta-analysis of prospective studies of red and processed meat intake and prostate cancer, Nutr J 9 (2010) 50.

[27] C. Rodriguez, S. J. Freedland, A. Deka, E. J. Jacobs, M. L. McCullough, A. V. Patel, M. J. Thun, E. E. Calle, Body mass index, weight change, and risk of prostate cancer in the Cancer Prevention Study II Nutrition Cohort, Cancer Epidemiol. Biomarkers Prev. 16 (1) (2007) 63-69.

[28] S. Strum, D. Pogliano, What every doctor who treats male patients should know, PCRI Insights vol. 8, no. 2 (May 2005).

[29] G. L. Lu-Yao, P. C. Albertsen, D. F. Moore, W. Shih, Y. Lin, R. S. DiPaola, M. J. Barry, A. Zietman, M. O'Leary, E. Walker-Corkery, S. L. Yao, Outcomes of localized prostate cancer following conservative management, JAMA 302 (11) (2009) 1202-1209.

[30] G. Oster, L. Lamerato, A. G. Glass, K. E. Richert-Boe, A. Lopez, K. Chung, A. Richhariya, T. Dodge, G. G. Wolff, A. Balakumaran, J. Edelsberg, Natural history of skeletal-related events in patients with breast, lung, or prostate cancer and metastases to bone: a 15-year study in two large US health systems, Support Care Cancer 21 (12) (2013) 3279-3286.

[31] L. Ye, H. G. Kynaston, W. G. Jiang, Bone metastasis in prostate cancer: molecular and cellular mechanisms (Review), Int. J. Mol. Med. 20 (1) (2007) 103-111.

[32] C. L. Carrol, F. G. Sommer, J. E. McNeal, T. A. Stamey, The abnormal prostate: MR imaging at 1.5 T with histopathologic correlation, Radiology 163 (2) (1987) 521-525.

[33] J. E. McNeal, E. A. Redwine, F. S. Freiha, T. A. Stamey, Zonal distribution of prostatic adenocarcinoma. Correlation with histologic pattern and direction of spread, Am. J. Surg. Pathol. 12 (12) (1988) 897-906.

[34] T. A. Stamey, A. N. Donaldson, C. E. Yemoto, J. E. McNeal, S. Sozen, H. Gill, Histological and clinical findings in 896 consecutive prostates treated only with radical retropubic prostatectomy: epidemiologic significance of annual changes, J. Urol. 160 (6 Pt 2) (1998) 2412-2417.

[35] R. J. Cohen, B. A. Shannon, M. Phillips, R. E. Moorin, T. M. Wheeler, K. L. Garrett, Central zone carcinoma of the prostate gland: a distinct tumor type with poor prognostic features, J. Urol. 179 (5) (2008) 1762-1767.

[36] R. Chou, J. M. Croswell, T. Dana, C. Bougatsos, I. Blazina, R. Fu, K. Gleitsmann, H. C. Koenig, C. Lam, A. Maltz, J. B. Rugge, K. Lin, Screening for prostate cancer: a review of the evidence for the U.S. Preventive Services Task Force, Ann. Intern. Med. 155 (11) (2011) 762-771.

[37] G. L. Andriole, E. D. Crawford, R. L. Grubb, S. S. Buys, D. Chia, T. R. Church, M. N. Fouad, E. P. Gelmann, P. A. Kvale, D. J. Reding, J. L. Weissfeld, L. A. Yokochi, B. O'Brien, J. D. Clapp, J. M. Rathmell, T. L. Riley, R. B. Hayes, B. S. Kramer, G. Izmirlian, A. B. Miller, P. F. Pinsky, P. C. Prorok, J. K. Gohagan, C. D. Berg, Mortality results from a randomized Prostate-cancer screening trial, New England Journal of Medicine 360 (13) (2009) 1310-1319.

[38] F. H. Schröder, J. Hugosson, M. J. Roobol, T. L. Tammela, S. Ciatto, V. Nelen, M. Kwiatkowski, M. Lujan, H. Lilja, M. Zappa, L. J. Denis, F. Recker, A. Pez, L. Määttänen, C. H. Bangma, G. Aus, S. Carlsson, A. Villers, X. Rebillard, T. van der Kwast, P. M. Kujala, B. G. Blijenberg, U.-H. Stenman, A. Huber, K. Taari, M. Hakama, S. M. Moss, H. J. de Koning, A. Auvinen, Prostate-cancer mortality at 11 years of follow-up, New England Journal of Medicine 366 (11) (2012) 981-990. 
[39] J. Hugosson, S. Carlsson, G. Aus, S. Bergdahl, A. Khatami, P. Lodding, C. G. Pihl, J. Stranne, E. Holmberg, H. Lilja, Mortality results from the Göteborg randomised population-based prostate-cancer screening trial, Lancet Oncol. 11 (8) (2010) 725-732.

[40] A. Bourdoumis, A. G. Papatsoris, M. Chrisofos, E. Efstathiou, A. Skolarikos, C. Deliveliotis, The novel prostate cancer antigen 3 (PCA3) biomarker, Int Braz J Urol 36 (6) (2010) 665-668.

[41] R. Morgan, A. Boxall, A. Bhatt, M. Bailey, R. Hindley, S. Langley, H. C. Whitaker, D. E. Neal, M. Ismail, H. Whitaker, N. Annels, A. Michael, H. Pandha, Engrailed-2 (EN2): a tumor specific urinary biomarker for the early diagnosis of prostate cancer, Clin. Cancer Res. 17 (5) (2011) 1090-1098.

[42] J. Brenner, A. Chinnaiyan, S. Tomlins, ETS fusion genes in prostate cancer, in: D. J. Tindall (Ed.), Prostate Cancer, Vol. 16 of Protein Reviews, Springer New York, 2013, pp. 139-183.

[43] C. M. Hoeks, J. O. Barentsz, T. Hambrock, D. Yakar, D. M. Somford, S. W. Heijmink, T. W. Scheenen, P. C. Vos, H. Huisman, I. M. van Oort, J. A. Witjes, A. Heerschap, J. J. Futterer, Prostate cancer: multiparametric MR imaging for detection, localization, and staging, Radiology 261 (1) (2011) 46-66.

[44] C. M. Moore, A. Ridout, M. Emberton, The role of MRI in active surveillance of prostate cancer, Curr Opin Urol 23 (3) (2013) 261-267.

[45] M. Noguchi, T. A. Stamey, J. E. McNeal, C. M. Yemoto, Relationship between systematic biopsies and histological features of 222 radical prostatectomy specimens: lack of prediction of tumor significance for men with nonpalpable prostate cancer, J. Urol. 166 (1) (2001) 104-109.

[46] G. P. Haas, N. B. Delongchamps, R. F. Jones, V. Chandan, A. M. Serio, A. J. Vickers, M. Jumbelic, G. Threatte, R. Korets, H. Lilja, G. de la Roza, Needle biopsies on autopsy prostates: sensitivity of cancer detection based on true prevalence, J. Natl. Cancer Inst. 99 (19) (2007) 1484-1489.

[47] A. V. Taira, G. S. Merrick, R. W. Galbreath, H. Andreini, W. Taubenslag, R. Curtis, W. M. Butler, E. Adamovich, K. E. Wallner, Performance of transperineal template-guided mapping biopsy in detecting prostate cancer in the initial and repeat biopsy setting, Prostate Cancer Prostatic Dis. 13 (1) (2010) 71-77.

[48] N. B. Delongchamps, M. Peyromaure, A. Schull, F. Beuvon, N. Bouazza, T. Flam, M. Zerbib, N. Muradyan, P. Legman, F. Cornud, Prebiopsy magnetic resonance imaging and prostate cancer detection: comparison of random and targeted biopsies, J. Urol. 189 (2) (2013) 493-499.

[49] B. Turkbey, P. L. Choyke, Multiparametric MRI and prostate cancer diagnosis and risk stratification, Curr Opin Urol 22 (4) (2012) 310-315.

[50] J. O. Barentsz, J. Richenberg, R. Clements, P. Choyke, S. Verma, G. Villeirs, O. Rouviere, V. Logager, J. J. Futterer, ESUR prostate MR guidelines 2012, Eur Radiol 22 (4) (2012) 746-757.

[51] H. Hricak, G. C. Dooms, J. E. McNeal, A. S. Mark, M. Marotti, A. Avallone, M. Pelzer, E. C. Proctor, E. A. Tanagho, MR imaging of the prostate gland: normal anatomy, AJR Am J Roentgenol 148 (1987) 51-58.

[52] O. Akin, E. Sala, C. S. Moskowitz, K. Kuroiwa, N. M. Ishill, D. Pucar, P. T. Scardino, H. Hricak, Transition zone prostate cancers: features, detection, localization, and staging at endorectal MR imaging, Radiology 239 (3) (2006) 784-792.

[53] L. Wang, Y. Mazaheri, J. Zhang, N. M. Ishill, K. Kuroiwa, H. Hricak, Assessment of biologic aggressiveness of prostate cancer: correlation of MR signal intensity with Gleason grade after radical prostatectomy, Radiology 246 (1) (2008) $168-176$.

[54] A. P. Kirkham, M. Emberton, C. Allen, How good is MRI at detecting and characterising cancer within the prostate?, Eur. Urol. 50 (6) (2006) 1163-1174.

[55] L. E. Quint, J. S. Van Erp, P. H. Bland, S. H. Mandell, E. A. Del Buono, H. B. Grossman, G. M. Glazer, P. W. Gikas, Carcinoma of the prostate: MR images obtained with body coils do not accurately reflect tumor volume, AJR Am J Roentgenol 156 (3) (1991) 511-516. 
[56] M. Cruz, K. Tsuda, Y. Narumi, Y. Kuroiwa, T. Nose, Y. Kojima, A. Okuyama, S. Takahashi, K. Aozasa, J. O. Barentsz, H. Nakamura, Characterization of low-intensity lesions in the peripheral zone of prostate on pre-biopsy endorectal coil MR imaging, Eur Radiol 12 (2) (2002) 357-365.

[57] W. Liu, B. Turkbey, J. Senegas, S. Remmele, S. Xu, J. Kruecker, M. Bernardo, B. J. Wood, P. A. Pinto, P. L. Choyke, Accelerated T2 mapping for characterization of prostate cancer, Magn Reson Med 65 (5) (2011) 1400-1406.

[58] G. P. Liney, M. Lowry, L. W. Turnbull, D. J. Manton, A. J. Knowles, S. J. Blackband, A. Horsman, Proton MR T2 maps correlate with the citrate concentration in the prostate, NMR Biomed 9 (2) (1996) 59-64.

[59] G. P. Liney, L. W. Turnbull, M. Lowry, L. S. Turnbull, A. J. Knowles, A. Horsman, In vivo quantification of citrate concentration and water T2 relaxation time of the pathologic prostate gland using 1H MRS and MRI, Magn Reson Imaging 15 (10) (1997) 1177-1186.

[60] G. P. Liney, A. J. Knowles, D. J. Manton, L. W. Turnbull, S. J. Blackband, A. Horsman, Comparison of conventional single echo and multi-echo sequences with a fast spin-echo sequence for quantitative T2 mapping: application to the prostate, J Magn Reson Imaging 6 (4) (1996) 603-607.

[61] P. Gibbs, D. J. Tozer, G. P. Liney, L. W. Turnbull, Comparison of quantitative T2 mapping and diffusion-weighted imaging in the normal and pathologic prostate, Magn Reson Med 46 (6) (2001) 1054-1058.

[62] S. Verma, B. Turkbey, N. Muradyan, A. Rajesh, F. Cornud, M. A. Haider, P. L. Choyke, M. Harisinghani, Overview of dynamic contrast-enhanced MRI in prostate cancer diagnosis and management, AJR Am J Roentgenol 198 (6) (2012) $1277-1288$.

[63] I. Gribbestad, K. Gjesdal, G. Nilsen, S. Lundgren, M. Hjelstuen, A. Jackson, An introduction to dynamic contrastenhanced MRI in oncology, in: A. Jackson, D. Buckley, G. Parker (Eds.), Dynamic Contrast-Enhanced Magnetic Resonance Imaging in Oncology, Medical Radiology, Springer Berlin Heidelberg, 2005, pp. 1-22.

[64] A. R. Padhani, Dynamic contrast-enhanced MRI in clinical oncology: current status and future directions, J Magn Reson Imaging 16 (4) (2002) 407-422.

[65] P. Tofts, T1-weighted DCE imaging concepts: modelling, acquisition and analysis, in: Magneton Flash, Siemens, 2010.

[66] A. B. Rosenkrantz, A. Sabach, J. S. Babb, B. W. Matza, S. S. Taneja, F. M. Deng, Prostate cancer: comparison of dynamic contrast-enhanced MRI techniques for localization of peripheral zone tumor, AJR Am J Roentgenol 201 (3) (2013) W471-478.

[67] G. J. Jager, E. T. Ruijter, C. A. van de Kaa, J. J. de la Rosette, G. O. Oosterhof, J. R. Thornbury, S. H. Ruijs, J. O. Barentsz, Dynamic TurboFLASH subtraction technique for contrast-enhanced MR imaging of the prostate: correlation with histopathologic results, Radiology 203 (3) (1997) 645-652.

[68] J. K. Kim, S. S. Hong, Y. J. Choi, S. H. Park, H. Ahn, C. S. Kim, K. S. Cho, Wash-in rate on the basis of dynamic contrast-enhanced MRI: usefulness for prostate cancer detection and localization, J Magn Reson Imaging 22 (5) (2005) 639-646.

[69] H. P. Schlemmer, J. Merkle, R. Grobholz, T. Jaeger, M. S. Michel, A. Werner, J. Rabe, G. van Kaick, Can pre-operative contrast-enhanced dynamic MR imaging for prostate cancer predict microvessel density in prostatectomy specimens?, Eur Radiol 14 (2) (2004) 309-317.

[70] B. Zelhof, M. Lowry, G. Rodrigues, S. Kraus, L. Turnbull, Description of magnetic resonance imaging-derived enhancement variables in pathologically confirmed prostate cancer and normal peripheral zone regions, BJU Int. 104 (5) (2009) $621-627$.

[71] C. G. van Niekerk, J. A. Witjes, J. O. Barentsz, J. A. van der Laak, C. A. Hulsbergen-van de Kaa, Microvascularity in transition zone prostate tumors resembles normal prostatic tissue, Prostate 73 (5) (2013) 467-475.

[72] D. Le Bihan, E. Breton, D. Lallemand, M. L. Aubin, J. Vignaud, M. Laval-Jeantet, Separation of diffusion and perfusion in intravoxel incoherent motion MR imaging, Radiology 168 (2) (1988) 497-505. 
[73] D. M. Koh, D. J. Collins, Diffusion-weighted MRI in the body: applications and challenges in oncology, AJR Am J Roentgenol 188 (6) (2007) 1622-1635.

[74] T. A. Huisman, Diffusion-weighted imaging: basic concepts and application in cerebral stroke and head trauma, Eur Radiol 13 (10) (2003) 2283-2297.

[75] D. Le Bihan, E. Breton, D. Lallemand, P. Grenier, E. Cabanis, M. Laval-Jeantet, MR imaging of intravoxel incoherent motions: application to diffusion and perfusion in neurologic disorders, Radiology 161 (2) (1986) 401-407.

[76] R. Shimofusa, H. Fujimoto, H. Akamata, K. Motoori, S. Yamamoto, T. Ueda, H. Ito, Diffusion-weighted imaging of prostate cancer, J Comput Assist Tomogr 29 (2) (2005) 149-153.

[77] A. R. Padhani, Integrating multiparametric prostate MRI into clinical practice, Cancer Imaging 11 Spec No A (2011) 27-37.

[78] Y. J. Choi, J. K. Kim, N. Kim, K. W. Kim, E. K. Choi, K. S. Cho, Functional MR imaging of prostate cancer, Radiographics 27 (2007) 63-75.

[79] K. W. Doo, D. J. Sung, B. J. Park, M. J. Kim, S. B. Cho, Y. W. Oh, Y. H. Ko, K. S. Yang, Detectability of low and intermediate or high risk prostate cancer with combined T2-weighted and diffusion-weighted MRI, Eur Radiol 22 (8) (2012) 1812-1819.

[80] T. Hambrock, D. M. Somford, H. J. Huisman, I. M. van Oort, J. A. Witjes, C. A. Hulsbergen-van de Kaa, T. Scheenen, J. O. Barentsz, Relationship between apparent diffusion coefficients at 3.0-T MR imaging and Gleason grade in peripheral zone prostate cancer, Radiology 259 (2) (2011) 453-461.

[81] Y. Itou, K. Nakanishi, Y. Narumi, Y. Nishizawa, H. Tsukuma, Clinical utility of apparent diffusion coefficient (ADC) values in patients with prostate cancer: can ADC values contribute to assess the aggressiveness of prostate cancer?, J Magn Reson Imaging 33 (1) (2011) 167-172.

[82] Y. Peng, Y. Jiang, C. Yang, J. Brown, T. Antic, I. Sethi, C. Schmid-Tannwald, M. Giger, S. Eggener, A. Oto, Quantitative analysis of multiparametric prostate MR images: differentiation between prostate cancer and normal tissue and correlation with Gleason score-a computer-aided diagnosis development study, Radiology 267 (1) (2013) 787-796.

[83] H. M. Awwad, J. Geisel, R. Obeid, The role of choline in prostate cancer, Clin. Biochem. 45 (18) (2012) 1548-1553.

[84] L. C. Costello, R. B. Franklin, The clinical relevance of the metabolism of prostate cancer; zinc and tumor suppression: connecting the dots, Mol. Cancer 5 (2006) 17.

[85] G. F. Giskeodegard, H. Bertilsson, K. M. Selnaes, A. J. Wright, T. F. Bathen, T. Viset, J. Halgunset, A. Angelsen, I. S. Gribbestad, M. B. Tessem, Spermine and citrate as metabolic biomarkers for assessing prostate cancer aggressiveness, PLoS ONE 8 (4) (2013) e62375.

[86] M. van der Graaf, R. G. Schipper, G. O. Oosterhof, J. A. Schalken, A. A. Verhofstad, A. Heerschap, Proton MR spectroscopy of prostatic tissue focused on the detection of spermine, a possible biomarker of malignant behavior in prostate cancer, MAGMA 10 (3) (2000) 153-159.

[87] S. Parfait, Classification de spectres et recherche de biomarqueurs en spectroscopie par résonqnce magnétique nulcléaire du proton dans les tumeurs prostatiques, Ph.D. thesis, Université de Bourgogne (2010).

[88] S. Verma, A. Rajesh, J. J. Futterer, B. Turkbey, T. W. Scheenen, Y. Pang, P. L. Choyke, J. Kurhanewicz, Prostate MRI and 3D MR spectroscopy: how we do it, AJR Am J Roentgenol 194 (6) (2010) 1414-1426.

[89] J. Scheidler, H. Hricak, D. B. Vigneron, K. K. Yu, D. L. Sokolov, L. R. Huang, C. J. Zaloudek, S. J. Nelson, P. R. Carroll, J. Kurhanewicz, Prostate cancer: localization with three-dimensional proton MR spectroscopic imaging-clinicopathologic study, Radiology 213 (2) (1999) 473-480.

[90] Y. Kaji, J. Kurhanewicz, H. Hricak, D. L. Sokolov, L. R. Huang, S. J. Nelson, D. B. Vigneron, Localizing prostate cancer in the presence of postbiopsy changes on MR images: role of proton MR spectroscopic imaging, Radiology 206 (3) (1998) $785-790$. 
[91] J. C. Vilanova, J. Comet, C. Barceló-Vidal, J. Barceló, E. López-Bonet, A. Maroto, M. Arzoz, À. Moreno, J. Areal, Peripheral zone prostate cancer in patients with elevated PSA levels and low free-to-total PSA ratio: detection with MR imaging and MR spectroscopy, Radiology 253 (1) (2009) 135-143.

[92] P. Walker, G. Crehange, S. Parfait, A. Cochet, P. Maignon, L. Cormier, F. Brunotte, Absolute quantification in 1H MRSI of the prostate at 3T, in: ISMRM Annual Meeting 2010, 2010.

[93] G. Lemaître, Absolute quantification at 3 T, Master's thesis, Université de Bourgogne, Heriot-Watt University, Universitat de Girona (2011).

[94] R. Nowak, Wavelet-based Rician noise removal for magnetic resonance imaging, Image Processing, IEEE Transactions on 8 (10) (1999) 1408-1419.

[95] J. V. Manjon, J. Carbonell-Caballero, J. J. Lull, G. Garcia-Marti, L. Marti-Bonmati, M. Robles, MRI denoising using non-local means, Med Image Anal 12 (4) (2008) 514-523.

[96] A. Buades, B. Coll, J. Morel, A review of image denoising algorithms, with a new one, Simul 4 (2005) 490-530.

[97] J. Mohan, V. Krishnaveni, Y. Guo, A survey on the magnetic resonance image denoising methods, Biomedical Signal Processing and Control 9 (0) (2014) $56-69$.

[98] S. Ozer, M. Haider, D. L. Langer, T. H. Van der Kwast, A. Evans, M. Wernick, J. Trachtenberg, I. Yetik, Prostate cancer localization with multispectral MRI based on Relevance Vector Machines, in: Biomedical Imaging: From Nano to Macro, 2009. ISBI '09. IEEE International Symposium on, 2009, pp. 73-76.

[99] S. Ozer, D. L. Langer, X. Liu, M. A. Haider, T. H. van der Kwast, A. J. Evans, Y. Yang, M. N. Wernick, I. S. Yetik, Supervised and unsupervised methods for prostate cancer segmentation with multispectral MRI, Med Phys 37 (4) (2010) 1873-1883.

[100] A. Pizurica, Image denoising using wavelets and spatial context modeling, Ph.D. thesis, Universiteit Gent (2002).

[101] D. Ampeliotis, A. Anonakoudi, K. Berberidis, E. Z. Psarakis, Computer aided detection of prostate cancer using fused information from dynamic contrast enhanced and morphological magnetic resonance images, in: IEEE International Conference on Signal Processing and Communications, 2007, pp. 888-891.

[102] D. Ampeliotis, A. Anonakoudi, K. Berberidis, E. Z. Psarakis, A. Kounoudes, A computer-aided system for the detection of prostate cancer based on magnetic resonance image analysis, in: International Symposium on Communications, Control and Signal Processing, 2008.

[103] S. Mallat, A wavelet tour of signal processing, Third Edition: The sparse way, 3rd Edition, Academic Press, 2008.

[104] R. Lopes, A. Ayache, N. Makni, P. Puech, A. Villers, S. Mordon, N. Betrouni, Prostate cancer characterization on MR images using fractal features, Med Phys 38 (1) (2011) 83-95.

[105] A. Pizurica, W. Philips, I. Lemahieu, M. Acheroy, A versatile wavelet domain noise filtration technique for medical imaging, IEEE Trans Med Imaging 22 (3) (2003) 323-331.

[106] D. Middleton, R. Esposito, Simultaneous optimum detection and estimation of signals in noise, Information Theory, IEEE Transactions on 14 (3) (1968) 434-444.

[107] M. Styner, C. Brechbuhler, G. Szckely, G. Gerig, Parametric estimate of intensity inhomogeneities applied to MRI, Medical Imaging, IEEE Transactions on 19 (3) (2000) 153-165.

[108] M. Jungke, W. Von Seelen, G. Bielke, S. Meindl, M. Grigat, P. Pfannenstiel, A system for the diagnostic use of tissue characterizing parameters in NMR-tomography, in: Proc. of Information Processing in Medical Imaging, Vol. 87, 1987, pp. 471-481.

[109] U. Vovk, F. Pernus, B. Likar, A review of methods for correction of intensity inhomogeneity in MRI, Medical Imaging, IEEE Transactions on 26 (3) (2007) 405-421.

[110] S. Viswanath, B. N. Bloch, M. Rosen, J. Chappelow, R. Toth, N. Rofsky, R. Lenkinski, E. Genega, A. Kalyanpur, A. Madabhushi, Integrating structural and functional imaging for computer assisted detection of prostate cancer on 
multi-protocol in vivo 3 Tesla MRI, in: Society of Photo-Optical Instrumentation Engineers (SPIE) Conference Series, Vol. 7260 of Society of Photo-Optical Instrumentation Engineers (SPIE) Conference Series, 2009.

[111] D. Lv, X. Guo, X. Wang, J. Zhang, J. Fang, Computerized characterization of prostate cancer by fractal analysis in MR images, J Magn Reson Imaging 30 (1) (2009) 161-168.

[112] A. Madabhushi, J. Udupa, A. Souza, Generalized scale: Theory, algorithms, and application to image inhomogeneity correction, Computer Vision and Image Understanding 101 (2) (2006) 100 - 121.

[113] L. G. Nyul, J. K. Udupa, On standardizing the MR image intensity scale, Magn Reson Med 42 (6) (1999) $1072-1081$.

[114] Y. Artan, D. Langer, M. Haider, T. H. Van der Kwast, A. Evans, M. Wernick, I. Yetik, Prostate cancer segmentation with multispectral MRI using cost-sensitive Conditional Random Fields, in: Biomedical Imaging: From Nano to Macro, 2009. ISBI '09. IEEE International Symposium on, 2009, pp. 278-281.

[115] Y. Artan, M. A. Haider, D. L. Langer, T. H. van der Kwast, A. J. Evans, Y. Yang, M. N. Wernick, J. Trachtenberg, I. S. Yetik, Prostate cancer localization with multispectral MRI using cost-sensitive support vector machines and conditional random fields, IEEE Trans Image Process 19 (9) (2010) 2444-2455.

[116] P. Liu, S. Wang, B. Turkbey, P. C. P. Grant, K.and Pinto, B. J. Wood, R. M. Summers, A prostate cancer computer-aided diagnosis system using multimodal magnetic resonance imaging and targeted biopsy labels, in: Proc. SPIE 8670, Medical Imaging 2013: Computer-Aided Diagnosis, 2013, pp. 86701G-86701G-6.

[117] L. G. Nyul, J. K. Udupa, X. Zhang, New variants of a method of MRI scale standardization, IEEE Trans Med Imaging 19 (2) (2000) 143-150.

[118] S. Viswanath, B. N. Bloch, J. Chappelow, P. Patel, N. Rofsky, R. Lenkinski, E. Genega, A. Madabhushi, Enhanced multiprotocol analysis via intelligent supervised embedding (EMPrAvISE): detecting prostate cancer on multi-parametric MRI, in: Proc. SPIE 7963, Medical Imaging 2011: Computer-Aided Diagnosis, 2011.

[119] S. E. Viswanath, N. B. Bloch, J. C. Chappelow, R. Toth, N. M. Rofsky, E. M. Genega, R. E. Lenkinski, A. Madabhushi, Central gland and peripheral zone prostate tumors have significantly different quantitative imaging signatures on 3 Tesla endorectal, in vivo T2-weighted MR imagery, J Magn Reson Imaging 36 (1) (2012) 213-224.

[120] A. Madabhushi, J. K. Udupa, New methods of MR image intensity standardization via generalized scale, Med Phys 33 (9) (2006) 3426-3434.

[121] E. Niaf, O. Rouvire, C. Lartizien, Computer-aided diagnosis for prostate cancer detection in the peripheral zone via multisequence MRI, in: Proc. SPIE 7963, Medical Imaging 2011: Computer-Aided Diagnosis, 2011.

[122] E. Niaf, O. Rouviere, F. Mege-Lechevallier, F. Bratan, C. Lartizien, Computer-aided diagnosis of prostate cancer in the peripheral zone using multiparametric MRI, Phys Med Biol 57 (12) (2012) 3833-3851.

[123] M. Wiart, L. Curiel, A. Gelet, D. Lyonnet, J. Y. Chapelon, O. Rouviere, Influence of perfusion on high-intensity focused ultrasound prostate ablation: a first-pass MRI study, Magn Reson Med 58 (1) (2007) 119-127.

[124] L. Chen, Z. Weng, L. Goh, M. Garland, An efficient algorithm for automatic phase correction of \{NMR\} spectra based on entropy minimization, Journal of Magnetic Resonance 158 (12) (2002) $164-168$.

[125] M. Osorio-Garcia, A. Croitor Sava, D. M. Sima, F. Nielsen, U. Himmelreich, S. Van Huffel, Magnetic Resonance Spectroscopy, InTech, 2012, Ch. Quantification improvements of 1H MRS Signals, pp. 1-27.

[126] S. Parfait, P. Walker, G. Crhange, X. Tizon, J. Mitran, Classification of prostate magnetic resonance spectra using Support Vector Machine, Biomedical Signal Processing and Control 7 (5) (2012) 499 - 508.

[127] H. Zhu, R. Ouwerkerk, P. B. Barker, Dual-band water and lipid suppression for MR spectroscopic imaging at 3 Tesla, Magn Reson Med 63 (6) (2010) 1486-1492.

[128] B. M. Kelm, B. H. Menze, C. M. Zechmann, K. T. Baudendistel, F. A. Hamprecht, Automated estimation of tumor probability in prostate magnetic resonance spectroscopic imaging: pattern recognition vs quantification, Magn Reson Med 57 (1) (2007) 150-159. 
[129] W. Pijnappel, A. van den Boogaart, R. de Beer, D. van Ormondt, SVD-based quantification of magnetic resonance signals, Journal of Magnetic Resonance (1969) 97 (1) (1992) 122 - 134.

[130] C. A. Lieber, A. Mahadevan-Jansen, Automated method for subtraction of fluorescence from biological Raman spectra, Appl Spectrosc 57 (11) (2003) 1363-1367.

[131] A. Devos, L. Lukas, J. A. Suykens, L. Vanhamme, A. R. Tate, F. A. Howe, C. Majos, A. Moreno-Torres, M. van der Graaf, C. Arus, S. Van Huffel, Classification of brain tumours using short echo time 1H MR spectra, J. Magn. Reson. 170 (1) (2004) 164-175.

[132] P. Tiwari, S. Viswanath, J. Kurhanewicz, A. Sridhar, A. Madabhushi, Multimodal wavelet embedding representation for data combination (MaWERiC): integrating magnetic resonance imaging and spectroscopy for prostate cancer detection, NMR Biomed 25 (4) (2012) 607-619.

[133] S. Ghose, A. Oliver, R. Marti, X. Llado, J. C. Vilanova, J. Freixenet, J. Mitra, D. Sidibe, F. Meriaudeau, A survey of prostate segmentation methodologies in ultrasound, magnetic resonance and computed tomography images, Comput Methods Programs Biomed 108 (1) (2012) 262-287.

[134] O. Chilali, A. Ouzzane, M. Diaf, N. Betrouni, A survey of prostate modeling for image analysis, Computers in Biology and Medicine 53 (0) (2014) $190-202$.

[135] L. Matulewicz, J. F. Jansen, L. Bokacheva, H. A. Vargas, O. Akin, S. W. Fine, A. Shukla-Dave, J. A. Eastham, H. Hricak, J. A. Koutcher, K. L. Zakian, Anatomic segmentation improves prostate cancer detection with artificial neural networks analysis of $1 \mathrm{H}$ magnetic resonance spectroscopic imaging, Journal of Magnetic Resonance Imaging (2013) n/a-n/a.

[136] P. Puech, N. Betrouni, N. Makni, A. S. Dewalle, A. Villers, L. Lemaitre, Computer-assisted diagnosis of prostate cancer using DCE-MRI data: design, implementation and preliminary results, Int J Comput Assist Radiol Surg 4 (1) (2009) $1-10$.

[137] P. C. Vos, T. Hambrock, J. O. Barenstz, H. J. Huisman, Combining T2-weighted with dynamic MR images for computerized classification of prostate lesions, in: Medical Imaging 2008: Computer-Aided Diagnosis, Vol. 6915, SPIE, 2008.

[138] P. C. Vos, T. Hambrock, C. A. Hulsbergen-van de Kaa, J. J. Futterer, J. O. Barentsz, H. J. Huisman, Computerized analysis of prostate lesions in the peripheral zone using dynamic contrast enhanced MRI, Med Phys 35 (3) (2008) $888-899$.

[139] G. J. S. Litjens, J. O. Barentsz, N. Karssemeijer, H. J. Huisman, Automated computer-aided detection of prostate cancer in MR images: from a whole-organ to a zone-based approach, in: Proc. SPIE 8315, Medical Imaging 2012: Computer-Aided Diagnosis, 2012, pp. 83150G-83150G-6.

[140] S. Klein, U. A. van der Heide, I. M. Lips, M. van Vulpen, M. Staring, J. P. Pluim, Automatic segmentation of the prostate in 3D MR images by atlas matching using localized mutual information, Med Phys 35 (4) (2008) 1407-1417.

[141] G. Litjens, O. Debats, W. van de Ven, N. Karssemeijer, H. Huisman, A pattern recognition approach to zonal segmentation of the prostate on MRI, Med Image Comput Comput Assist Interv 15 (Pt 2) (2012) 413-420.

[142] G. Litjens, O. Debats, J. Barentsz, N. Karssemeijer, H. Huisman, Computer-iided detection of prostate cancer in MRI, Medical Imaging, IEEE Transactions on 33 (5) (2014) 1083-1092.

[143] G. Litjens, R. Toth, W. van de Ven, C. Hoeks, S. Kerkstra, B. van Ginneken, G. Vincent, G. Guillard, N. Birbeck, J. Zhang, R. Strand, F. Malmberg, Y. Ou, C. Davatzikos, M. Kirschner, F. Jung, J. Yuan, W. Qiu, Q. Gao, P. E. Edwards, B. Maan, F. van der Heijden, S. Ghose, J. Mitra, J. Dowling, D. Barratt, H. Huisman, A. Madabhushi, Evaluation of prostate segmentation algorithms for MRI: the PROMISE12 challenge, Med Image Anal 18 (2) (2014) $359-373$

[144] T. R. Langerak, U. A. van der Heide, A. N. Kotte, M. A. Viergever, M. van Vulpen, J. P. Pluim, Label fusion in atlasbased segmentation using a selective and iterative method for performance level estimation (SIMPLE), IEEE Trans Med Imaging 29 (12) (2010) 2000-2008. 
[145] S. Viswanath, B. N. Bloch, E. Genega, N. Rofsky, R. Lenkinski, J. Chappelow, R. Toth, A. Madabhushi, A comprehensive segmentation, registration, and cancer detection scheme on 3 Tesla in vivo prostate DCE-MRI, Med Image Comput Comput Assist Interv 11 (Pt 1) (2008) 662-669.

[146] R. Toth, J. Chappelow, M. Rosen, S. Pungavkar, A. Kalyanpur, A. Madabhushi, Multi-attribute non-initializing texture reconstruction based active shape model (MANTRA), Med Image Comput Comput Assist Interv 11 (Pt 1) (2008) 653661.

[147] T. F. Cootes, C. J. Taylor, D. H. Cooper, J. Graham, Active shape models\&Mdash;Their training and application, Comput. Vis. Image Underst. 61 (1) (1995) 38-59.

[148] G. J. S. Litjens, P. C. Vos, J. O. Barentsz, N. Karssemeijer, H. J. Huisman, Automatic computer aided detection of abnormalities in multi-parametric prostate MRI, in: Proc. SPIE 7963, Medical Imaging 2011: Computer-Aided Diagnosis, 2011, pp. 79630T-79630T-7.

[149] P. C. Vos, J. O. Barentsz, N. Karssemeijer, H. J. Huisman, Automatic computer-aided detection of prostate cancer based on multiparametric magnetic resonance image analysis, Phys Med Biol 57 (6) (2012) 1527-1542.

[150] H. Huisman, P. Vos, G. Litjens, T. Hambrock, J. Barentsz, Computer aided detection of prostate cancer using T2, DWI and DCE MRI: methods and clinical applications, in: Proceedings of the 2010 international conference on Prostate cancer imaging: computer-aided diagnosis, prognosis, and intervention, MICCAI'10, Springer-Verlag, Berlin, Heidelberg, 2010 , pp. $4-14$

[151] P. Tiwari, M. Rosen, A. Madabhushi, A hierarchical spectral clustering and nonlinear dimensionality reduction scheme for detection of prostate cancer from magnetic resonance spectroscopy (MRS), Med Phys 36 (9) (2009) 3927-3939.

[152] J. Shi, J. Malik, Normalized cuts and image segmentation, Pattern Analysis and Machine Intelligence, IEEE Transactions on 22 (8) (2000) 888-905.

[153] J. B. Maintz, M. A. Viergever, A survey of medical image registration, Med Image Anal 2 (1) (1998) 1-36.

[154] B. Zitová, J. Flusser, Image registration methods: a survey, Image and Vision Computing 21 (11) (2003) 977 - 1000.

[155] J. Mitra, R. Marti, A. Oliver, X. Llado, J. C. Vilanova, F. Meriaudeau, A comparison of thin-plate splines with automatic correspondences and B-splines with uniform grids for multimodal prostate registration, in: Society of Photo-Optical Instrumentation Engineers (SPIE) Conference Series, Vol. 7964 of Society of Photo-Optical Instrumentation Engineers (SPIE) Conference Series, 2011.

[156] J. Mitra, Z. Kato, R. Marti, A. Oliver, X. Llado, D. Sidibe, S. Ghose, J. C. Vilanova, J. Comet, F. Meriaudeau, A spline-based non-linear diffeomorphism for multimodal prostate registration, Med Image Anal 16 (6) (2012) 1259-1279.

[157] R. Toth, S. Doyle, S. Pungavkar, A. Kalyanpur, A. Madabhushi, A boosted ensemble scheme for accurate landmark detection for active shape models, in: SPIE Medical Imaging, Vol. 7260, Orlando, FL, 2009.

[158] J. Pluim, J. Maintz, M. Viergever, Mutual-information-based registration of medical images: a survey, IEEE Transcations on Medical Imaging 22 (8) (2003) 986-1004.

[159] J. Chappelow, B. N. Bloch, N. Rofsky, E. Genega, R. Lenkinski, W. DeWolf, A. Madabhushi, Elastic registration of multimodal prostate MRI and histology via multiattribute combined mutual information, Med Phys 38 (4) (2011) 20052018.

[160] R. H. Byrd, P. Lu, J. Nocedal, C. Zhu, A limited memory algorithm for bound constrained optimization, SIAM J. Sci. Comput. 16 (5) (1995) 1190-1208.

[161] P. Viola, W. M. Wells, III, Alignment by maximization of mutual information, Int. J. Comput. Vision 24 (2) (1997) $137-154$.

[162] J. Mitra, Multimodal image registration applied to magnetic resonance and ultrasound prostatic images, Ph.D. thesis, Universitat de Girona and Université de Bourgogne (2012).

[163] V. Giannini, A. Vignati, S. Mazzetti, M. De Luca, C. Bracco, M. Stasi, F. Russo, E. Armando, D. Regge, A prostate 
CAD system based on multiparametric analysis of DCE T1-w, and DW automatically registered images, in: Proc. SPIE 8670, Medical Imaging 2013: Computer-Aided Diagnosis, 2013, pp. 86703E-86703E-6.

[164] P. C. Vos, T. Hambrock, J. O. Barenstz, H. J. Huisman, Computer-assisted analysis of peripheral zone prostate lesions using T2-weighted and dynamic contrast enhanced T1-weighted MRI, Phys Med Biol 55 (6) (2010) 1719-1734.

[165] D. Rueckert, L. I. Sonoda, C. Hayes, D. L. Hill, M. O. Leach, D. J. Hawkes, Nonrigid registration using free-form deformations: application to breast MR images, IEEE Trans Med Imaging 18 (8) (1999) 712-721.

[166] X. Liu, D. L. Langer, M. A. Haider, Y. Yang, M. N. Wernick, I. S. Yetik, Prostate cancer segmentation with simultaneous estimation of Markov random field parameters and class, IEEE Trans Med Imaging 28 (6) (2009) 906-915.

[167] S. Mazzetti, M. De Luca, C. Bracco, A. Vignati, V. Giannini, M. Stasi, F. Russo, E. Armando, S. Agliozzo, D. Regge, A CAD system based on multi-parametric analysis for cancer prostate detection on DCE-MRI, in: Proc. SPIE 7963, Medical Imaging 2011: Computer-Aided Diagnosis, 2011, pp. 79633Q-79633Q-7.

[168] Y. S. Sung, H. J. Kwon, B. W. Park, G. Cho, C. K. Lee, K. S. Cho, J. K. Kim, Prostate cancer detection on dynamic contrast-enhanced MRI: computer-aided diagnosis versus single perfusion parameter maps, AJR Am J Roentgenol 197 (5) (2011) 1122-1129.

[169] P. Tiwari, A. Madabhushi, M. Rosen, A hierarchical unsupervised spectral clustering scheme for detection of prostate cancer from magnetic resonance spectroscopy (MRS), Med Image Comput Comput Assist Interv 10 (Pt 2) (2007) 278-286.

[170] P. Tiwari, M. Rosen, A. Madabhushi, Consensus-locally linear embedding (C-LLE): application to prostate cancer detection on magnetic resonance spectroscopy, Med Image Comput Comput Assist Interv 11 (Pt 2) (2008) 330-338.

[171] P. Tiwari, M. Rosen, G. Reed, J. Kurhanewicz, A. Madabhushi, Spectral embedding based probabilistic boosting tree (ScEPTre): classifying high dimensional heterogeneous biomedical data, Med Image Comput Comput Assist Interv 12 (Pt 2) (2009) 844-851.

[172] P. Tiwari, J. Kurhanewicz, M. Rosen, A. Madabhushi, Semi supervised multi kernel (SeSMiK) graph embedding: identifying aggressive prostate cancer via magnetic resonance imaging and spectroscopy, Med Image Comput Comput Assist Interv 13 (Pt 3) (2010) 666-673.

[173] P. Tiwari, J. Kurhanewicz, A. Madabhushi, Multi-kernel graph embedding for detection, Gleason grading of prostate cancer via MRI/MRS, Med Image Anal 17 (2) (2013) 219-235.

[174] S. Viswanath, P. Tiwari, M. Rosen, A. Madabhushi, A meta-classifier for detecting prostate cancer by quantitative integration of In Vivo magnetic resonance spectroscopy and magnetic resonance imaging, in: Medical Imaging 2008: Computer-Aided Diagnosis, Vol. 6915, SPIE, 2008.

[175] D. L. Langer, T. H. van der Kwast, A. J. Evans, J. Trachtenberg, B. C. Wilson, M. A. Haider, Prostate cancer detection with multi-parametric MRI: logistic regression analysis of quantitative T2, diffusion-weighted imaging, and dynamic contrast-enhanced MRI, J Magn Reson Imaging 30 (2) (2009) 327-334.

[176] J. Prewitt, Picture processing and psychohistories, Academic Press, 1970, Ch. Object enhancement and extraction.

[177] I. Sobel, Camera models and machine perception, Tech. rep., DTIC Document (1970).

[178] R. Kirsch, Computer determination of the constituent structure of biological images, Computers and Biomedical Research 4 (3) (1971) $315-328$.

[179] D. Gabor, Theory of communication. Part 1: The analysis of information, Electrical Engineers - Part III: Radio and Communication Engineering, Journal of the Institution of 93 (26) (1946) 429-441.

[180] J. G. Daugman, Uncertainty relation for resolution in space, spatial frequency, and orientation optimized by twodimensional visual cortical filters, J Opt Soc Am A 2 (7) (1985) 1160-1169.

[181] R. Haralick, K. Shanmugam, I. Dinstein, Textural features for image classification, Systems, Man and Cybernetics, IEEE Transactions on SMC-3 (6) (1973) 610-621.

[182] T. Antic, Y. Peng, Y. Jiang, M. L. Giger, S. Eggener, A. Oto, A study of T2-weighted MR image texture features and 
diffusion-weighted MR image features for computer-aided diagnosis of prostate cancer, in: Proc. SPIE 8670, Medical Imaging 2013: Computer-Aided Diagnosis, 2013, pp. 86701H-86701H-6.

[183] A. Benassi, S. Cohen, J. Istas, Identifying the multifractional function of a Gaussian process, Statistics \& Probability Letters 39 (4) (1998) $337-345$.

[184] N. Ahmed, T. Natarajan, K. Rao, Discrete cosine transform, Computers, IEEE Transactions on C-23 (1) (1974) 90-93.

[185] T. Leung, J. Malik, Representing and recognizing the visual appearance of materials using three-dimensional textons, International Journal of Computer Vision 43 (1) (2001) 29-44.

[186] N. Dalal, B. Triggs, Histograms of oriented gradients for human detection, in: Computer Vision and Pattern Recognition, 2005. CVPR 2005. IEEE Computer Society Conference on, Vol. 1, 2005, pp. 886-893 vol. 1.

[187] S. Belongie, J. Malik, J. Puzicha, Shape matching and object recognition using shape contexts, Pattern Analysis and Machine Intelligence, IEEE Transactions on 24 (4) (2002) 509-522.

[188] G. Zhao, T. Ahonen, J. Matas, M. Pietikainen, Rotation-Invariant Image and Video Description With Local Binary Pattern Features, Image Processing, IEEE Transactions on 21 (4) (2012) 1465-1477.

[189] T. Ojala, M. Pietikäinen, D. Harwood, A comparative study of texture measures with classification based on featured distributions, Pattern Recognition 29 (1) (1996) 51-59.

[190] P. S. Tofts, Modeling tracer kinetics in dynamic Gd-DTPA MR imaging, J Magn Reson Imaging 7 (1) (1997) 91-101.

[191] P. Castorina, P. P. Delsanto, C. Guiot, Classification scheme for phenomenological universalities in growth problems in physics and other sciences, Phys. Rev. Lett. 96 (2006) 188701.

[192] H. Ratiney, M. Sdika, Y. Coenradie, S. Cavassila, D. van Ormondt, D. Graveron-Demilly, Time-domain semi-parametric estimation based on a metabolite basis set, NMR Biomed 18 (1) (2005) 1-13.

[193] L. Vanhamme, A. van den Boogaart, S. Van Huffel, Improved method for accurate and efficient quantification of MRS data with use of prior knowledge, J. Magn. Reson. 129 (1997) 35-45.

[194] T. Coleman, Y. Li, An interior trust region approach for nonlinear minimization subject to bounds, Tech. rep., Cornell University (1993).

[195] S. W. Provencher, Estimation of metabolite concentrations from localized in vivo proton NMR spectra, Magn Reson Med 30 (6) (1993) 672-679.

[196] R. Coifman, M. Wickerhauser, Entropy-based algorithms for best basis selection, Information Theory, IEEE Transactions on 38 (2) (1992) 713-718.

[197] Y. Saeys, I. Inza, P. Larranaga, A review of feature selection techniques in bioinformatics, Bioinformatics 23 (19) (2007) $2507-2517$.

[198] H. Peng, F. Long, C. Ding, Feature selection based on mutual information criteria of max-dependency, max-relevance, and min-redundancy, Pattern Analysis and Machine Intelligence, IEEE Transactions on 27 (8) (2005) $1226-1238$.

[199] I. Fodor, A survey of dimension reduction techniques (2002).

[200] I. T. Jolliffe, Principal Component Analysis, 2nd Edition, Springer, 2002.

[201] M. Belkin, P. Niyogi, Laplacian eigenmaps and spectral techniques for embedding and clustering, in: Advances in Neural Information Processing Systems 14, MIT Press, 2001, pp. 585-591.

[202] S. T. Roweis, L. K. Saul, Nonlinear dimensionality reduction by locally linear embedding, Science 290 (5500) (2000) $2323-2326$

[203] C. M. Bishop, Pattern recognition and machine learning, Springer-Verlag New York, Inc., Secaucus, NJ, USA, 2006.

[204] A. Fred, A. Jain, Combining multiple clusterings using evidence accumulation, Pattern Analysis and Machine Intelligence, IEEE Transactions on 27 (6) (2005) 835-850.

[205] J. Friedman, Regularized discriminant analysis, Journal of the American Statistical Association 84 (405) (1989) pp. $165-175$. 
[206] I. Rish, An empirical study of the naive Bayes classifier, in: IJCAI 2001 workshop on empirical methods in artificial intelligence, Vol. 3, 2001, pp. 41-46.

[207] Y. Freund, R. Schapire, A decision-theoretic generalization of on-line learning and an application to boosting, Journal of Computer and System Sciences 55 (1) (1997) 119 - 139.

[208] L. Breiman, Random forests, Machine Learning 45 (1) (2001) 5-32.

[209] Z. Tu, Probabilistic boosting-tree: learning discriminative models for classification, recognition, and clustering, in: Computer Vision, 2005. ICCV 2005. Tenth IEEE International Conference on, Vol. 2, 2005, pp. 1589-1596 Vol. 2.

[210] J. Friedman, T. Hastie, R. Tibshirani, Additive logistic regression: a statistical view of boosting, Annals of Statistics 28 (1998) 2000.

[211] C. Rasmussen, C. Williams, Gaussian processes for machine learning, The MIT Press, 2005.

[212] V. Vapnik, A. Lerner, Pattern Recognition using Generalized Portrait Method, Automation and Remote Control 24.

[213] B. E. Boser, I. M. Guyon, V. N. Vapnik, A training algorithm for optimal margin classifiers, in: Proceedings of the Fifth Annual Workshop on Computational Learning Theory, COLT '92, ACM, New York, NY, USA, 1992, pp. 144-152.

[214] M. Tipping, Sparse Bayesian learning and the relevance vector machine, Journal of Machine Learning Research 1 (2001) $211-244$.

[215] J. Quinonero-Candela, A. Girard, C. Rasmussen, Prediction at an Uncertain Input for Gaussian processes and relevance vector machines application to Multiple-Step ahead time-series forecasting, Tech. rep., DTU Informatics (2002).

[216] D. F. Specht, Probabilistic neural networks for classification, mapping, or associative memory, in: Neural Networks, 1988., IEEE International Conference on, 1988, pp. 525-532 vol.1.

[217] B. Efron, Estimating the error rate of a prediction rule: Improvement on cross-validation, Journal of the American Statistical Association 78 (382) (1983) pp. 316-331.

[218] C. E. Metz, Receiver operating characteristic analysis: a tool for the quantitative evaluation of observer performance and imaging systems, J Am Coll Radiol 3 (6) (2006) 413-422. 
Table 1: Overview of the features associated with each MRI modality used for medical diagnosis by radiologists. Acronyms: Prostate Cancer (CaP) - Signal Intensity (SI) - Gleason Score (GS).

\begin{tabular}{|c|c|c|c|c|}
\hline Modality & Significant features & $\mathrm{CaP}$ & Healthy tissue & $\begin{array}{c}\text { GS } \\
\text { correlation }\end{array}$ \\
\hline $\mathrm{T}_{2}-\mathrm{W} \mathrm{MRI}$ & $\begin{array}{l}\text { SI } \\
\text { Shape } \\
--------\ldots---\cdot- \\
\text { SI } \\
\text { Shape }\end{array}$ & $\begin{array}{c}\text { low-SI in PZ }[51] \\
\text { round or ill-defined mass in } \\
\text { PZ }[8] \\
\text { low-SI in CG }[52,50] \\
\text { homogeneous mass with } \\
\text { ill-defined edges in CG }[52,50]\end{array}$ & $\begin{array}{l}\text { intermediate to high-SI in PZ [51] } \\
\text { low-SI in CG }[52,50]\end{array}$ & $+[53]$ \\
\hline $\mathrm{T}_{2}$ map & SI & low-SI $[58,61]$ & intermediate to high-SI $[58,61]$ & $+[57,58,59]$ \\
\hline \multirow[t]{2}{*}{ DCE MRI } & $\begin{array}{l}\text { Semi-quantitative features [62]: } \\
\text { - wash-in } \\
\text { - wash-out } \\
\text { - integral under the curve } \\
\text { - maximum signal intensity } \\
\text { - time-to-peak enhancement }\end{array}$ & $\begin{array}{l}\text { faster } \\
\text { faster } \\
\text { higher } \\
\text { higher } \\
\text { faster }\end{array}$ & $\begin{array}{l}\text { slower } \\
\text { slower } \\
\text { lower } \\
\text { lower } \\
\text { slower }\end{array}$ & $\begin{array}{l}0 \\
0 \\
0 \\
0 \\
0\end{array}$ \\
\hline & $\begin{array}{l}\text { Quantitative features (Tofts' } \\
\text { parameters }[65] \text { ): } \\
-\mathrm{k}_{\mathrm{ep}} \\
-\mathrm{K}^{\mathrm{trans}}\end{array}$ & $\begin{array}{l}\text { higher } \\
\text { higher }\end{array}$ & $\begin{array}{l}\text { lower } \\
\text { lower }\end{array}$ & $\begin{array}{l}0 \\
0\end{array}$ \\
\hline DW MRI & SI & higher-SI $[74,50]$ & lower-SI $[74,50]$ & + \\
\hline ADC map & SI & low-SI [50] & high-SI [50] & $+[80,81,82]$ \\
\hline MRSI & $\begin{array}{l}\text { Metabolites: } \\
\text { Citrate (2.64 ppm) [88] } \\
\text { Choline (3.21 ppm) [88] } \\
\text { Spermine (3.11 ppm) [88] }\end{array}$ & $\begin{array}{l}\text { lower concentration }[83,84,86] \\
\text { higher concentration }[83,84,86] \\
\text { lower concentration }[83,84,86]\end{array}$ & $\begin{array}{l}\text { higher concentration }[83,84,86] \\
\text { lower concentration }[83,84,86] \\
\text { higher concentration }[83,84,86]\end{array}$ & $\begin{array}{l}+[85] \\
0[85] \\
+[85]\end{array}$ \\
\hline
\end{tabular}

Notes:

$+=$ significantly correlated.

$0=$ no correlation. 
Table 2: Overview of the different studies reviewed with their main characteristics. Acronyms: number (\#) - image regularization (Img. Reg.).

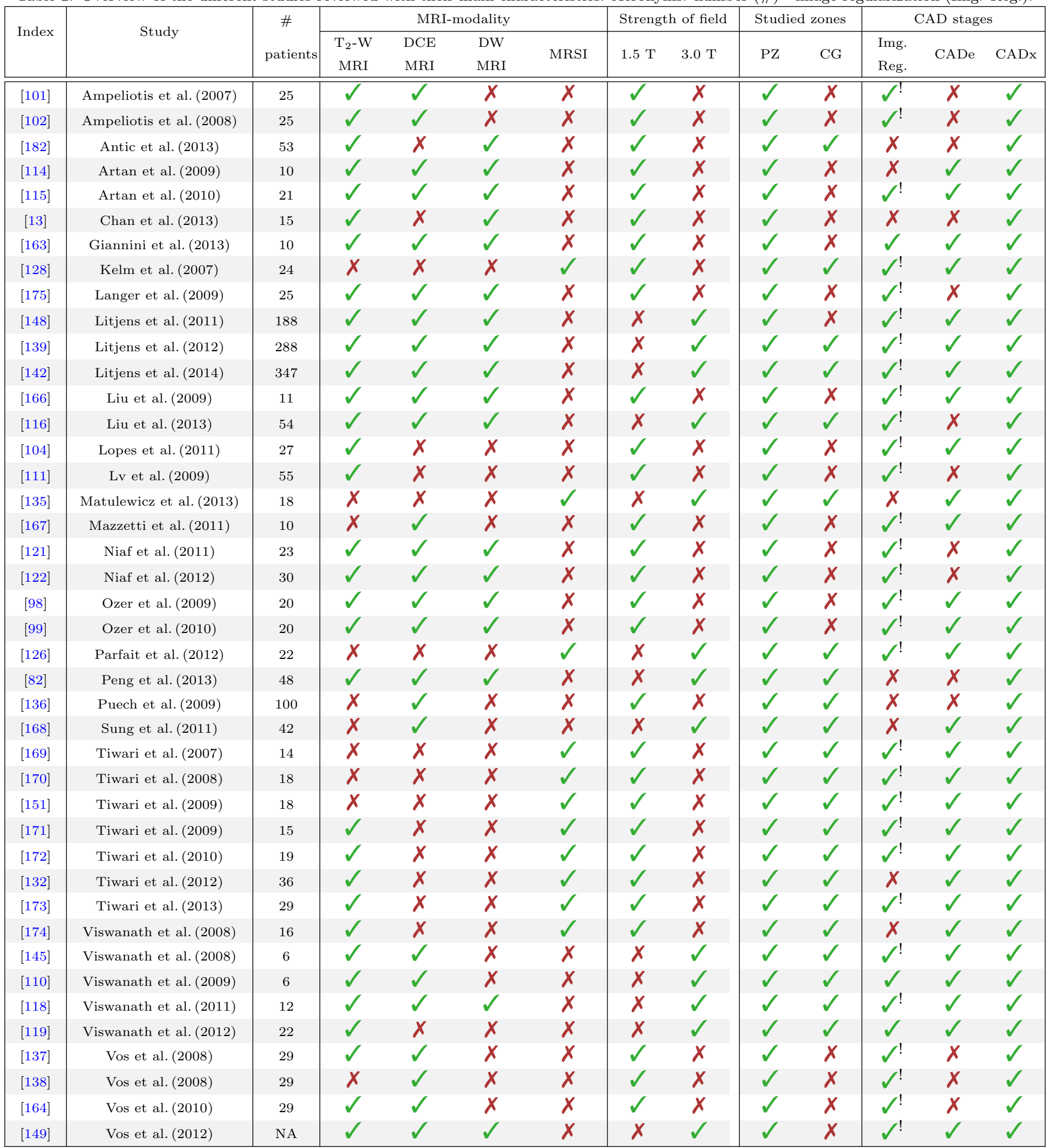

Notes:

$X$ : not used or not implemented.

$\Omega^{!}$: partially implemented.

$\sqrt{ }$ : used or implemented. 


\section{Pre-processing operations}

\section{References}

MRI pre-processing:

Noise filtering:

$\begin{array}{ll}\text { Median filtering } & {[98,99]} \\ \text { Wavelet-based filtering } & {[101,102,104]}\end{array}$

Bias correction:

$\begin{array}{lc}\text { Parametric methods } & {[111,110]} \\ \text { Non-parametric methods } & {[118]}\end{array}$

Standardization:

Statistical-based normalization:

$[114,115,111,98,99,110$, $118,119]$

Organ SI-based normalization

$[121,122]$

MRSI pre-processing:

Phase correction

Water and lipid residuals filtering

Baseline correction

[126, 132]

Frequency alignment

Normalization

[126]

Table 4: Overview of the segmentation methods used in CAD systems.

\section{Segmentation methods}

MRI-based segmentation:

Manual segmentation

Region-based segmentation

Model-based segmentation

MRSI-based segmentation:

Clustering

\section{References}

$[114,115,135,121,122,98$, 99, 136, 137, 138, 164, 149] [139, 142]

[148, 145, 110, 118, 149]

[151] 
Table 5: Classification of the different registration methods used in the CAD systems reviewed. Acronyms: gradient descent (GD), Nelder-Mead (NM).

\begin{tabular}{|c|c|c|c|c|c|c|c|c|c|}
\hline \multirow{2}{*}{$\begin{array}{l}\text { Study } \\
\text { index }\end{array}$} & \multirow{2}{*}{$\begin{array}{l}\text { Modality } \\
\text { registered }\end{array}$} & \multirow{2}{*}{ Type } & \multicolumn{2}{|c|}{ Geometric model } & \multicolumn{3}{|c|}{ Similarity measure } & \multicolumn{2}{|c|}{ Optimizer } \\
\hline & & & Affine & Elastic & MSE & MI & $\mathrm{CMI}$ & GD & L-BFGS-B \\
\hline$[101,102]$ & $\mathrm{T}_{2}-\mathrm{W}-\mathrm{DCE}$ & $2 \mathrm{D}$ & $\sqrt{ }$ & - & $\sqrt{ }$ & - & - & - & - \\
\hline$[163]$ & $\mathrm{T}_{2}-\mathrm{W}-\mathrm{DW}$ & $2 \mathrm{D}$ & $\sqrt{ }$ & $\mathscr{V}$ & - & - & - & - & - \\
\hline [163] & $\mathrm{T}_{2}-\mathrm{W}-\mathrm{DCE}$ & $2 \mathrm{D}$ & $\checkmark$ & $\mathscr{V}$ & - & $\sqrt{ }$ & - & $\checkmark$ & - \\
\hline$[145,110]$ & $\mathrm{T}_{2}-\mathrm{W}-\mathrm{DCE}$ & $2 \mathrm{D}$ & $\checkmark$ & - & - & $\sqrt{ }$ & - & - & - \\
\hline$[118]$ & $\mathrm{T}_{2}-\mathrm{W}-\mathrm{DCE}-\mathrm{DW}$ & $3 \mathrm{D}$ & $\mathscr{V}$ & - & - & - & $\mathscr{}$ & $\sqrt{ }$ & - \\
\hline$[137]$ & $\mathrm{T}_{2}-\mathrm{W}-\mathrm{DCE}$ & $3 \mathrm{D}$ & $\sqrt{ }$ & - & - & $\mathscr{V}$ & - & - & - \\
\hline$[164]$ & $\mathrm{T}_{2}-\mathrm{W}-\mathrm{DCE}$ & $3 \mathrm{D}$ & $\checkmark$ & $\sqrt{ }$ & - & $\mathscr{V}$ & - & - & $\checkmark$ \\
\hline
\end{tabular}

Notes:

-: not used or not mentioned.

$\sqrt{ }$ : used or implemented.

Table 6: Overview of the CADe strategies employed in CAD systems.

CADe: ROIs selection strategy

All voxels-based approach

Lesions candidate detection

\section{References}

$[114,115,163,128,166,104$,

$135,167,98,99,126,168$,

169, 170, 151, 171, 172, 132,

173, 174, 145, 110, 118, 119]

[148, 139, 142, 149] 


\section{MRI image:}

Voxel-wise detection

Intensity-based

Edge-based

Prewitt operator

Sobel operator

Kirsch operator

Gabor filtering

Texture-based

Haralick features

Fractal analysis

DCT

Wavelet-based features

Gaussian filter bank

Position-based

Region-wise detection

Statistical-based

Percentiles

Statistical-moments

Histogram-based

PDF

HOG

Shape context

LBP

Anatomical-based

\section{DCE signal:}

Whole spectra approach

Semi-quantitative approach

Quantitative approach

Toft model

Brix model

Weibull function

PUM

\section{MRSI signal:}

Whole spectra approach Quantification approach Wavelet-based approach

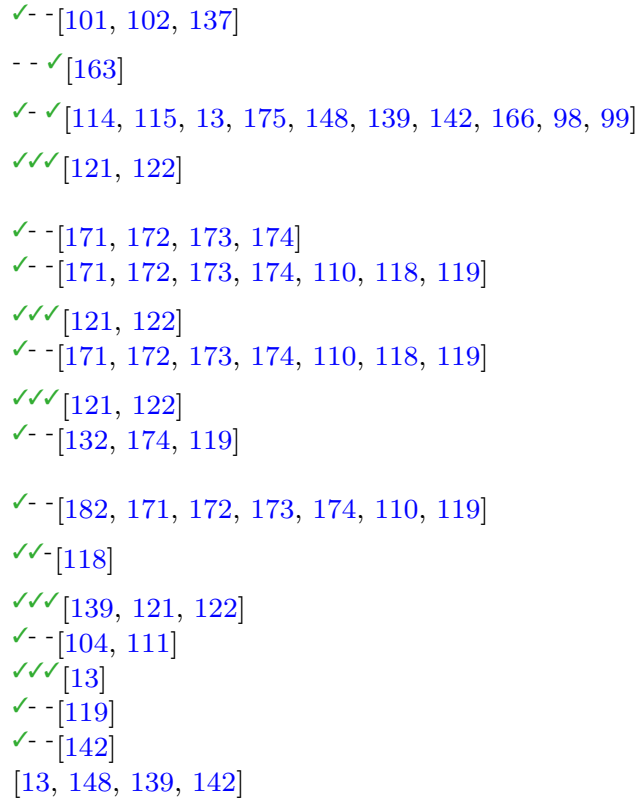

$-\checkmark-[138]$

- $\checkmark[182,82]$

$\checkmark \checkmark-[164]$

$\checkmark \checkmark \checkmark[148,139,142,121,122,149]$

$\checkmark-$ - $[101,102,171,172,173,174,110,119]$

- - $[182]$

$\checkmark \checkmark-[118]$

$\checkmark-\checkmark[82]$

$\checkmark \checkmark \checkmark[148,139,142,121,122]$

$\checkmark \checkmark \checkmark[116]$

$\checkmark \checkmark \checkmark[116]$

$\checkmark \checkmark \checkmark[116]$

$\checkmark \checkmark \checkmark[116]$

$[139,142,135]$

[101, 102]

$\checkmark$ ! 136$]$

$[167,121,122,168]$

$\checkmark^{!}[116 ?]$

$[163,175,148,139,142,167,121,122]$

$\checkmark ! 114,115,98,99]$

$[166,168]$

$[163,167]$

$[163,167]$

\section{Notes:}

$(\checkmark|-\checkmark|-\checkmark \mid-)$ : triplet stating the implementation or not of the feature for respectively $\mathrm{T}_{2}$-W-MRI images, DCE-MRI images, DW-MRI images.

$\checkmark$ : used or implemented.

$\checkmark$ !: partially implemented. 
Table 8: Parameters used as features for a DCE semi-quantitative analysis in CAD systems

\section{Semi-quantitative features \\ Explanations}

Amplitude features:

$S_{0}$
$S_{\max }$
$S_{p}$
$S_{f}$

Time features:

$t_{0}$
$t_{\max }$
$t_{p}$
$t_{f}$
$t_{t p}$

Derivatives and integral features:

$W I$

$W O$

$I A U C$
Amplitude at the onset of the enhancement

Amplitude corresponding to $95 \%$ of the maximum amplitude

Amplitude corresponding to the maximum amplitude

Amplitude at the final time point

Time at the onset of the enhancement

Time corresponding to $95 \%$ of the maximum amplitude

Time corresponding to the maximum amplitude

Final time

Time to peak which is the time from $t_{0}$ to $t_{p}$

Wash-in rate corresponding to the signal slope from $t_{0}$ to $t_{m}$ or $t_{p}$ Wash-out rate corresponding to the signal slope from $t_{m}$ or $t_{p}$ to $t_{p}$ Initial area under the curve which is the area between $t_{0}$ to $t_{f}$

Table 9: Overview of the feature selection and extraction methods used in CAD systems.

\section{Dimension reduction methods}

Feature selection:

Statistical test

MI-based methods

Feature extraction:

Linear mapping

PCA

Non-linear mapping

Laplacian eigenmaps

LLE and LLE-based
[121, 122, 149

[121, 122, 137]

$[170,151]$

\section{References}

$[169,171,151,172,174,118]$

$[170,151,145,174]$ 


\begin{tabular}{|c|c|}
\hline Classifier & References \\
\hline Rule-based method: & {$[111,136]$} \\
\hline \multicolumn{2}{|l|}{ Clustering methods: } \\
\hline$k$-means clustering & {$[169,170,151]$} \\
\hline$k-\mathrm{NN}$ & {$[139,121,122]$} \\
\hline \multicolumn{2}{|l|}{ Linear model classifiers: } \\
\hline LDA & {$[182,13,142,121,122,149]$} \\
\hline Logistic regression & {$[128,175]$} \\
\hline \multicolumn{2}{|l|}{ Non-linear classifier: } \\
\hline QDA & {$[119]$} \\
\hline \multicolumn{2}{|l|}{ Probabilistic classifier: } \\
\hline Naive Bayes & {$[163,167,121,122]$} \\
\hline \multicolumn{2}{|l|}{ Ensemble learning classifiers: } \\
\hline AdaBoost & {$[142,104]$} \\
\hline Random forest & {$[128,142,132,173,110]$} \\
\hline Probabilistic boosting tree & {$[151,172,132]$} \\
\hline \multicolumn{2}{|l|}{ Kernel method: } \\
\hline Gaussian processes & {$[128]$} \\
\hline \multicolumn{2}{|l|}{ Sparse kernel methods: } \\
\hline SVM & $\begin{array}{l}{[114,115,13,148,139,116,104,} \\
121,122,98,99,126,82,168,132,\end{array}$ \\
\hline RVM & $\begin{array}{l}137,138,164,149] \\
{[98,99]}\end{array}$ \\
\hline \multicolumn{2}{|l|}{ Neural network: } \\
\hline Multiple layer perceptron & {$[135,126]$} \\
\hline Probabilistic neural network & {$[101,102,118]$} \\
\hline \multicolumn{2}{|l|}{ Graphical model classifiers: } \\
\hline Markov random field & {$[166,99]$} \\
\hline Conditional random field & {$[114,115]$} \\
\hline
\end{tabular}




\section{Model validation techniques}

LOOCV

$k$-CV

\section{References}

$[101,102,182,114,115,13,163,128,139$, $142,167,121,122,98,99,82,136,173$ $118,137,137,164]$

$[148,126,151,171,172,132,119,110$ $149]$

Table 12: Overview of the evaluation metrics used in CAD systems.

\begin{tabular}{ll}
\hline Evaluation metrics & References \\
\hline Accuracy & {$[114,115,166,168,132]$} \\
Sensitivity - Specificity & {$[114,115,163,166,104,167,98,99,126$,} \\
& $82,170,151,174,145]$ \\
ROC - AUC & {$[102,182,13,163,128,175,116,104,111$,} \\
& $135,167,121,122,82,171,172,132,173$, \\
& $110,118,119,137,138,164]$ \\
FROC & {$[148,139,149]$} \\
Dice's coefficient & {$[114,115,166,98]$} \\
\hline
\end{tabular}




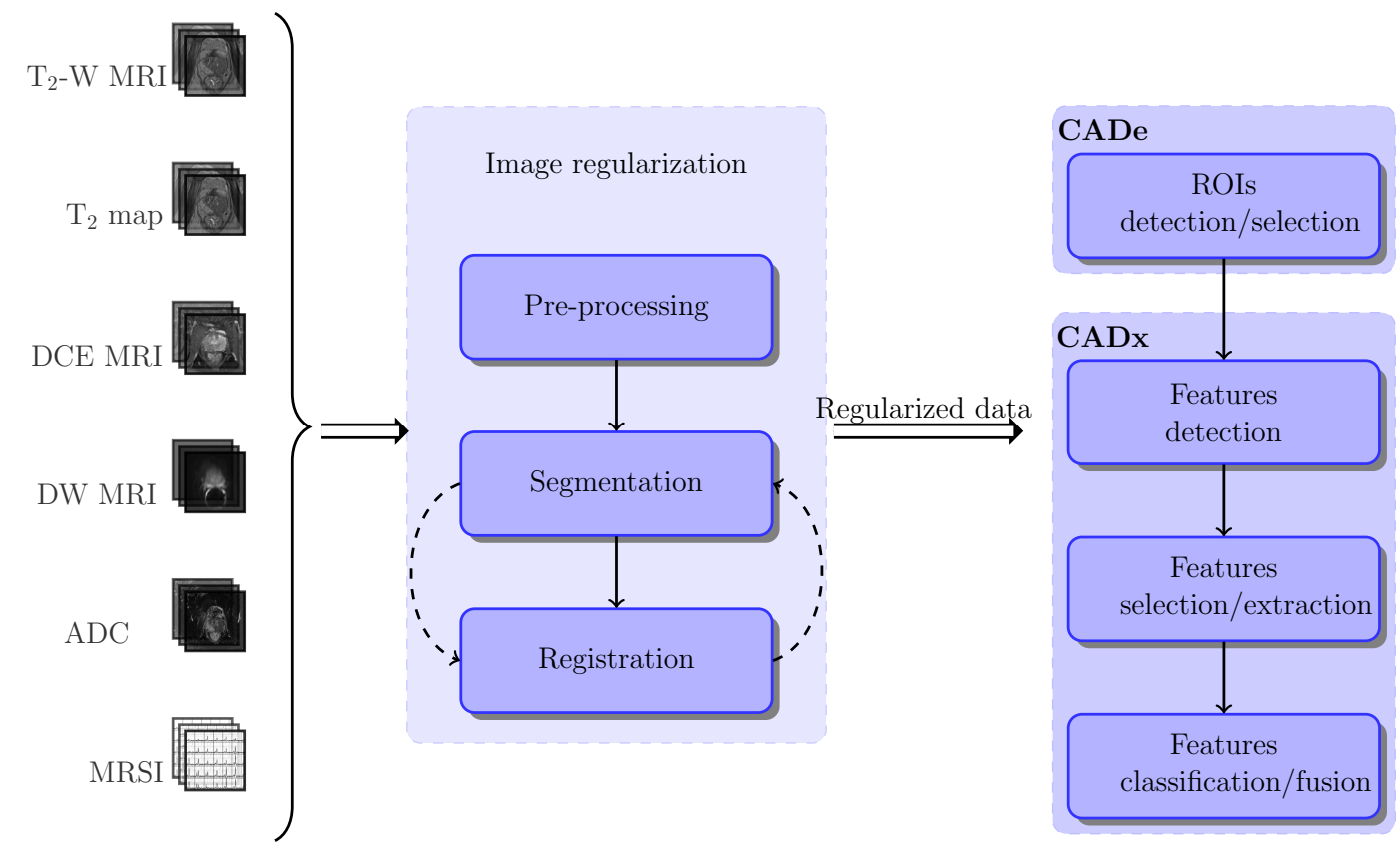

Figure 1: Common CAD framework based on MRI images used to detect CaP.

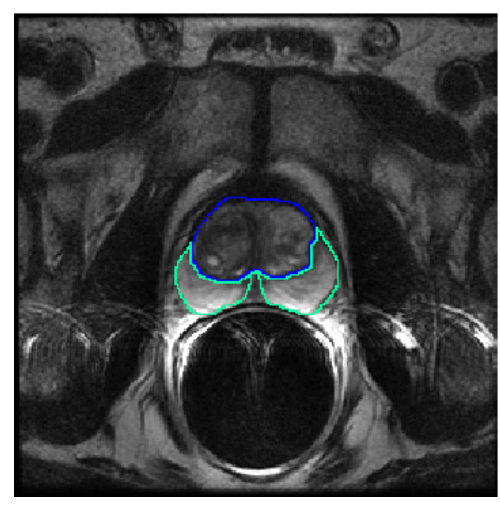

(a) $\mathrm{T}_{2}$-W-MRI slice of an healthy prostate acquire with a 1.5 Tesla MRI. The blue contour represents the CG while the $\mathrm{PZ}$ corresponds to the green contour.

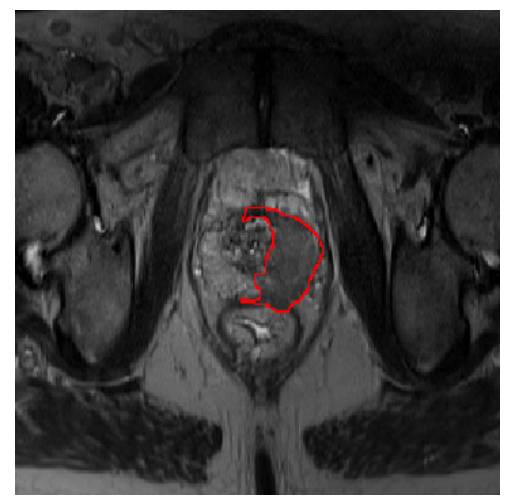

(b) $\mathrm{T}_{2}$-W-MRI slice of a prostate with a CaP highlighted in the $\mathrm{PZ}$ using a 3.0 Tesla MRI scanner.

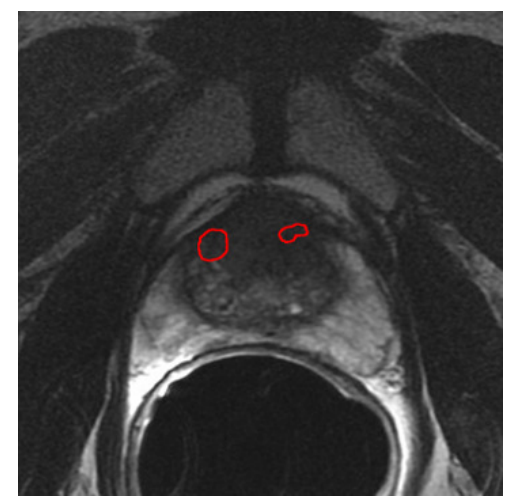

(c) $\mathrm{T}_{2}$-W-MRI slice of a prostate with a CaP highlighted in the CG using a 3.0 Tesla MRI scanner.

Figure 2: Rendering of $\mathrm{T}_{2}$-W-MRI prostate image with both 1.5 and 3.0 Tesla MRI scanner. 


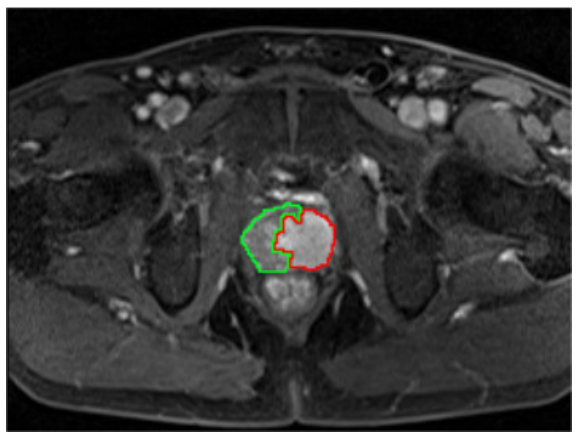

(a)

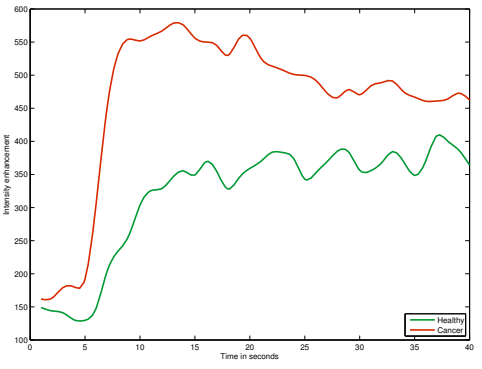

(b)

Figure 3: Illustration of: (a) $\mathrm{T}_{1}-\mathrm{W}-\mathrm{MRI}$ image and (b) typical enhancement signals observed in DCE-MRI analysis collected with a 3.0 Tesla MRI scanner. The red curve is typical from CaP while the green curve is characteristic of healthy tissue.

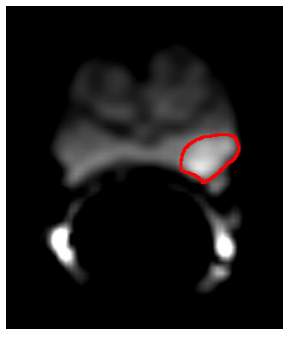

(a)

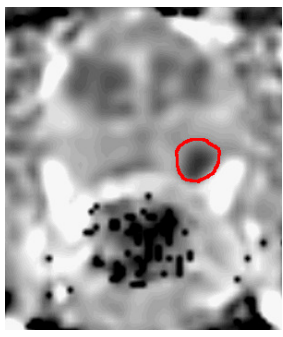

(b)

Figure 4: Illustration of: (a) DW-MRI and (b) ADC map. The signal intensity corresponding to cancer are inversely correlated on these two types of imaging techniques. The cancer is highlighted in red.

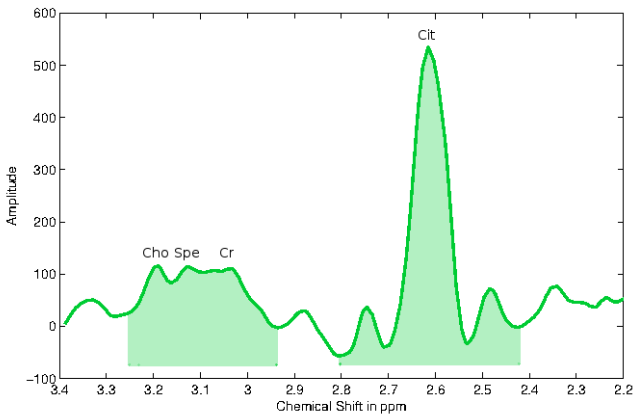

(a)

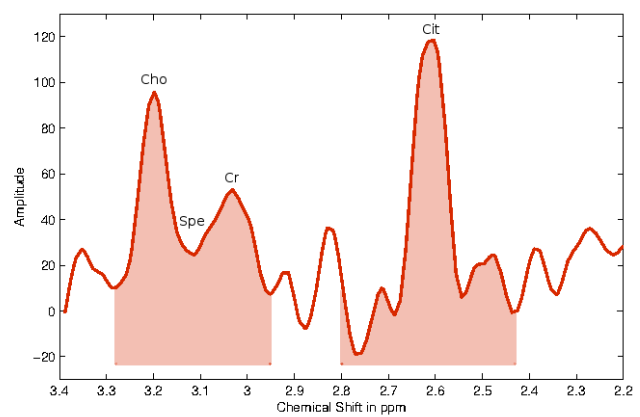

(b)

Figure 5: Illustration of an MRSI spectrum both (a) healthy and (b) cancerous voxel with a 3.0 Tesla MRI. The highlighted areas corresponds to the related concentration of the metabolites which is computed by integrating the area under each peak. Acronyms: Choline (Cho), Spermine (Spe), Creatine (Cr) and Citrate (Cit). 


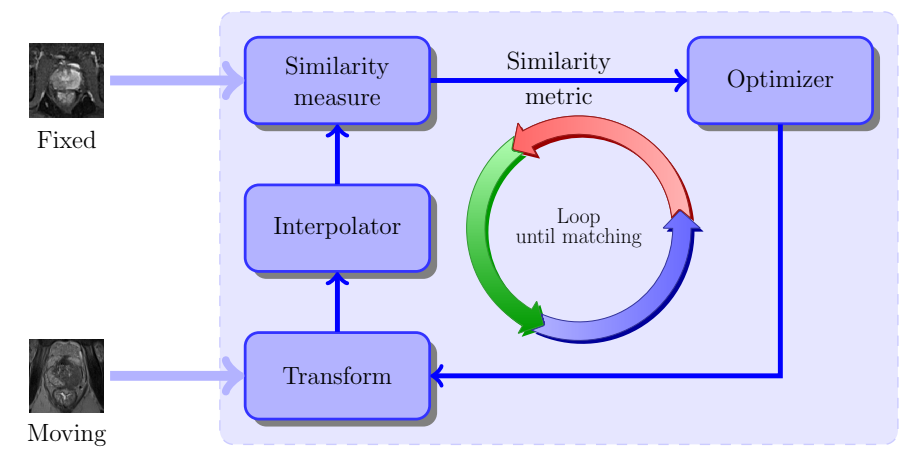

Figure 6: Typical framework involved to solve the registration problem.

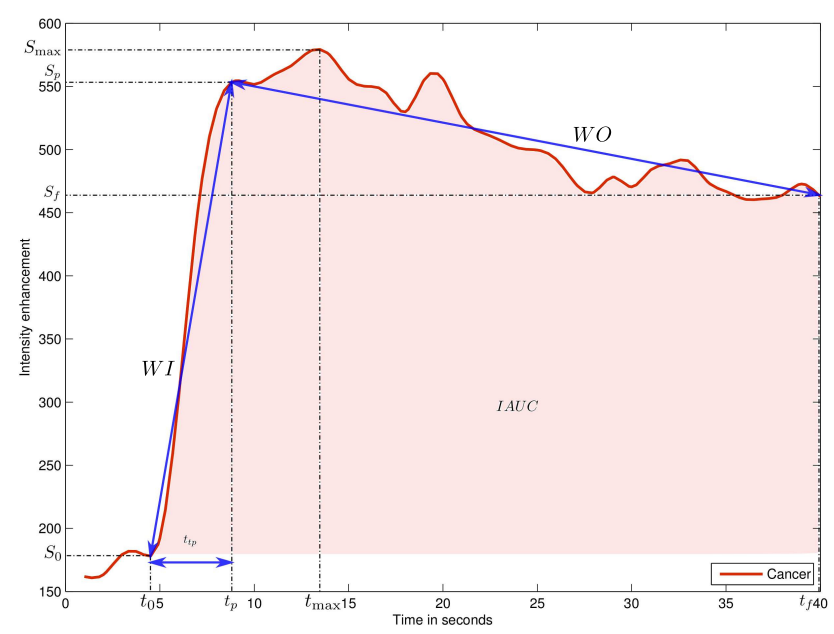

Figure 7: Graphical representation of the different semi-quantitative features used for DCE-MRI analysis.

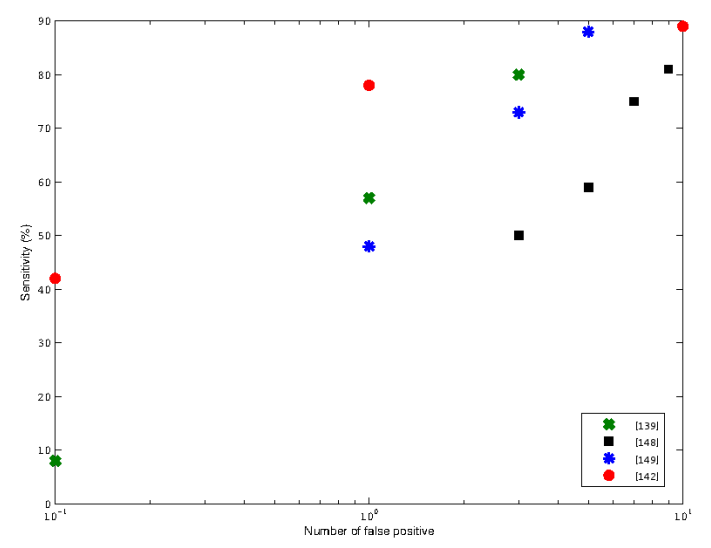

Figure 8: Comparison in terms of FROC of the methods using data from 3.0 Tesla MRI scanner. 


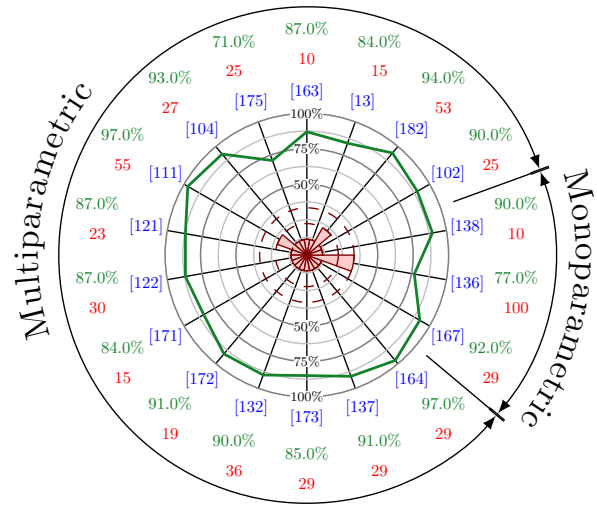

(a)

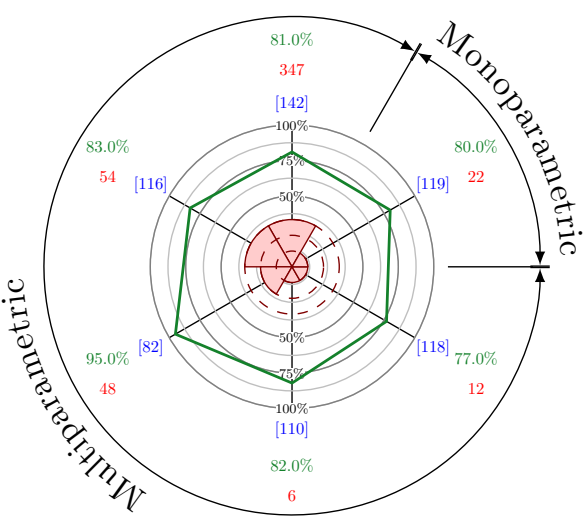

(b)

Figure 9: Numerical and graphical comparison of the results in terms of AUC for 1.5 and 3.0 Tesla MRI scanners. The green value represents the metric and are graphically reported in the green curve in the center of the figure. The red value and areas correspond to the number of patients in the dataset. The numbers between brackets in blue correspond to the reference as reported in Table 2 .

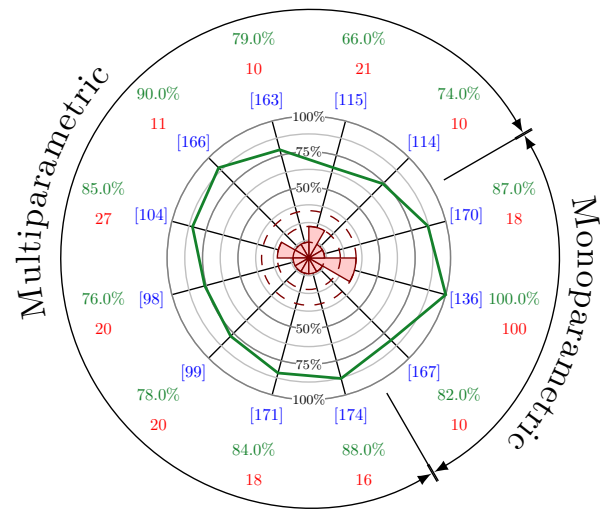

(a)

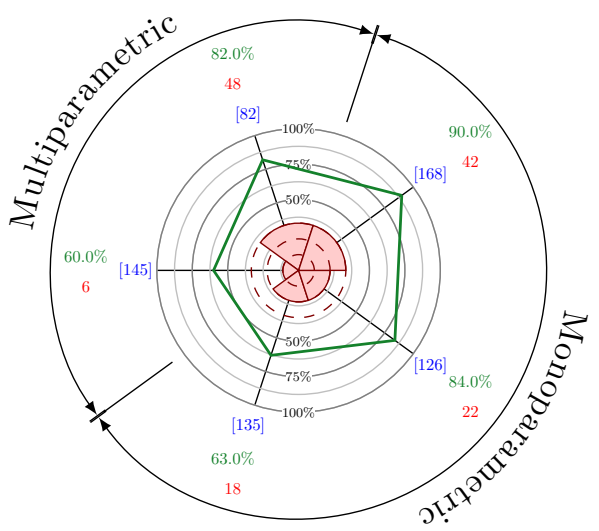

(c)

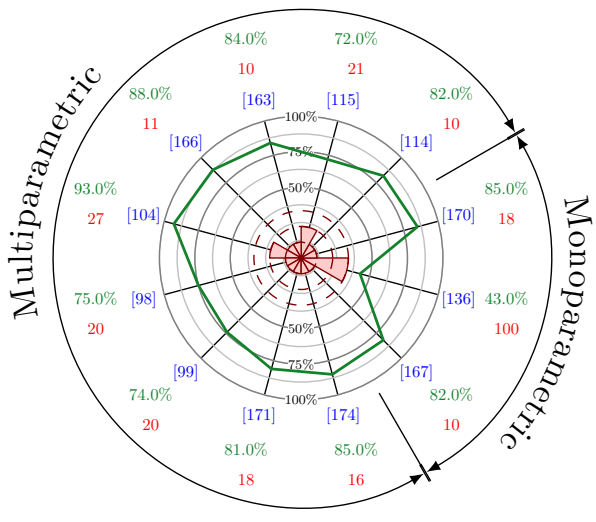

(b)

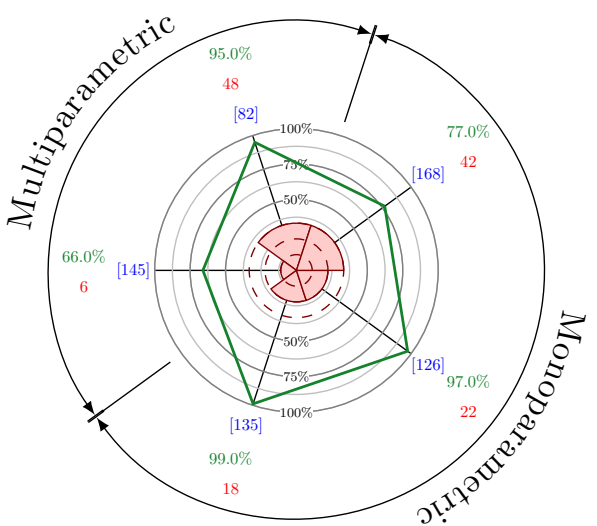

(d)

Figure 10: Numerical and graphical comparison of the results in terms of sensitivity (a), (c) and specificity (b), (d) for 1.5 and 3.0 Tesla MRI scanners. The value in green represents the metric and are graphically reported in the green curve in the center of the figure. The red value and areas correspond to the number of patients in the dataset. The numbers between brackets in blue correspond to the reference as reported in Table 2. 